\title{
THE ALGEBRAIC STRUCTURE OF RELATIVISTIC WAVE EQUATIONS
}

\author{
A. CANT and C. A. HURST
}

(Received 17 April 1978)

(Revised 5 July 1978)

\begin{abstract}
The algebraic structure of relativistic wave equations of the form

$$
\left(\alpha^{\mu} \partial_{\mu}+i \kappa\right) \psi(x)=0
$$

is considered. This leads to the problem of finding all Lie algebras $L$ which contain the Lorentz Lie algebra so $(3,1)$ and also contain a "four-vector" $\alpha^{\mu}$; such an $L$ gives rise to a family of wave equations. The simplest possibility is the Bhabha equations where $L \cong s o(5)$. Some authors have claimed that this is the only one, but it is shown that there are many other possibilities still in accord with physical requirements. Known facts about representations, along with Dynkin's theory of the embeddings of Lie algebras, are used to obtain a partial classification of wave equations. The discrete transformations $C, P, T$ are also discussed, along with reality properties. Finally, a simple example of a family of wave equations based on $L=s p(12)$ is considered in detail. The $s o(3,1)$ content and mass spectra are given for the low order members of the family, and the problem of causality is briefly discussed.
\end{abstract}

\section{Introduction}

In this paper we shall consider the problem of describing all the finite dimensional Lorentz invariant first-order wave equations of the form

$$
\left(\alpha^{\mu} \frac{\partial}{\partial x^{\mu}}+i \kappa I_{n}\right) \psi(x)=0,
$$

where the $\alpha^{\mu}(\mu=0,1,2,3)$ are $n \times n$ matrices. We take $\kappa$ to be a real non-zero constant throughout the paper, except at the end of Section 3, where we briefly consider the case where $\kappa$ is also an $n \times n$ matrix.

This problem has been considered by many authors, notably Dirac [1], Fierz and Pauli [2], Bhabha [3], Harish-Chandra [4], Le Couteur [5] and Gel'fand and 
Yaglom [6]. We refer the reader to the books by Corson [7], and Gel'fand, Minlos and Shapiro [8] for further details. The equations given by Dirac, Fierz and Pauli involve subsidiary conditions on the components of $\psi(x)$, and lead to the wellknown difficulties involved in the introduction of an external field. We follow Bhabha in assuming that there are no such subsidiary conditions; a given wave equation then in general describes particles with a spectrum of rest masses and spins [3].

Let us recall some of the properties of the wave equation (1.1). Under a Lorentz transformation $x^{\prime}=\Lambda x$ we suppose that

$$
\psi^{\prime}\left(x^{\prime}\right)=\pi(\Lambda) \psi(x),
$$

where $\pi(\Lambda)$ is some $n \times n$ matrix. As is well known, $\pi$ is a representation of the Lorentz group $S O(3,1)$, and so the generators $I_{\mu \nu}\left(I_{\mu \nu}=-I_{v \mu}\right)$ of $\pi$ must satisfy the commutation relations

$$
\left[I_{\mu v}, I_{\rho \sigma}\right]=g_{v \rho} I_{\mu \sigma}-g_{\mu \rho} I_{v \sigma}-g_{v \sigma} I_{\mu \rho}+g_{\mu \sigma} I_{v \rho} .
$$

Here we have taken $g_{00}=-g_{11}=-g_{22}=-g_{33}=1$, and $g_{\mu v}=0$ if $\mu \neq v$. The condition that $(1.1)$ is invariant is

or

$$
\pi^{-1}(\Lambda) \alpha^{\mu} \pi(\Lambda)=\Lambda_{v}^{\mu} \alpha^{\nu}
$$

$$
\left[I_{\mu v}, \alpha_{\rho}\right]=g_{v \rho} \alpha_{\mu}-g_{\mu \rho} \alpha_{v} .
$$

This means that $\left\{\alpha^{\mu}\right\}$ transforms like a four-vector operator.

Let us denote by $S$ the Lie algebra generated by the $I_{\mu \nu}$ and $\alpha_{\rho}$. It is important to observe that the commutators $\left[\alpha_{\mu}, \alpha_{v}\right]$ are not determined by any direct physical considerations. We note that it is quite consistent with (1.3) to assume that $\left[\alpha_{\mu}, \alpha_{v}\right]=c I_{\mu v}(c \in \mathrm{C})$, and so that $S$ is the Lie algebra of $S O(3,2)$. This is the most familiar case, the simplest wave equation of this type being the Dirac equation, and it was worked out in detail by Bhabha [3]. It was asserted by Bauer [9] that this case is essentially the only one possible. More recently, Lorente, Huddleston and Roman [10] claimed that, for a large class of representation $\pi, S=s p(4, \mathbf{R}) \cong$ $s o(3,2)$. However, these claims are incorrect: $\dagger$ counter-examples are easily found, and so more general possibilities for $S$ must be considered. We may formulate the problem as follows:

"Find all Lie algebras $L$ which contain a copy of so $(3,1)$, and also contain a fourvector operator $\left\{\alpha^{\mu}\right\} . L$ is to be minimal in the sense that $L$ is generated by $s o(3,1)$ and the $\alpha^{\mu}$."

Given such a Lie algebra $L$, we obtain a whole family of wave equations by taking all the irreducible representations of $L$. The Lorentz content of these equa-

$\dagger$ Reference [10] was shown to be incorrect by Bracken in [29]. 
tions is obtained by finding the branching rule for the reduction of $L$ to $\operatorname{so}(3,1)$, while the possible values of the rest mass are simply related to the eigenvalues of the matrix $\alpha^{0}$ in these representations. The family of wave equations associated with the irreducible representations of $L$ can be regarded as the wave equations for the states of a quark model, the quark being the multiplet associated with the fundamental representation. These higher representations are constructed by group theoretical procedures analogous to the construction of hadrons from quarks.

The main aim of this paper is to present some of these more general possibilities, in order to systematize the results already found $[3,6,11]$ for the general structure of $\alpha^{\mu}$. We have given invariant proofs wherever possible. In Section 2, we formulate the problem more precisely, giving the notation which will be in force throughout the paper. We discuss briefly those aspects of Dynkin's theory of semi-simple sub-algebras of semi-simple Lie algebras which we need [12]. Also, we give a more elegant way of describing a "vector operator" such as $\left\{\alpha^{\mu}\right\}$.

In Section 3, we give a general analysis of the Lie algebra structure of wave equations. Section 4 contains a description of the discrete transformations: charge conjugation, space reflection and time reversal. We also discuss the existence of an invariant Hermitian form, which is required in order that the wave equation (1.1) be obtainable from an invariant Lagrangian.

Next, in Section 5, we consider an example in detail: we shall call this example the "Kursunoğlu equation" (in fact it is a special case of the wave equation recently proposed by Kursunoğlu [13]). We give the so(3,1)content for the lowest dimensional representations of this equation, their corresponding mass spectra, and discuss the external field problem for this case. Section 6 presents some conclusions.

\section{Summary of Dynkin's theory. Tensor operators}

Since this paper is concerned with the problem of specifying the embedding of one Lie algebra in another, and since the branching rules depend on the embedding, we give here a brief account of Dynkin's general theory [12,14].

We note first that, although in the Introduction we mentioned Lie algebras $L$ over the real field $\mathbf{R}$, in fact we need only consider their complexifications $L^{\mathbf{c}}$. This is so because the finite dimensional irreducible representations of $L$ are in a one-to-one correspondence with the finite dimensional irreducible representations of $L^{C}$. Thus, for example, instead of $s o(3,1)$, we work with its complexification $s o(4, C) \cong s l(2, C) \oplus s l(2, C)$. When it is necessary to distinguish the factors in this direct sum, we write them as $\operatorname{sl}(2, \mathrm{C})$, and $\operatorname{sl}(2, \mathrm{C})^{\prime}$.

Our notation will mainly follow that of Humphreys [15]. Let $L$ be a complex semi-simple Lie algebra of rank $l$. Choosing a Cartan sub-algebra (CSA) $H$ of $L$, with dual space $H^{*}$, we let $\Phi$ denote the set of roots relative to $H$. If $\Delta=\left\{\alpha_{1}, \ldots, \alpha_{1}\right\}$ 
is a basis of $\Phi$, we denote by $\Phi^{+}$the set of positive roots relative to $\Delta$, and write $\Lambda^{+} \subset H^{*}$ for the set of dominant integral linear functions on $H$ (see [15], p. 112). Any $\lambda \in \Lambda^{+}$can be written in the form

$$
\lambda=\sum_{i=1}^{l} m_{i} \lambda_{i},
$$

where $m_{i} \in \mathbf{Z}^{+}$, and $\lambda_{1}, \ldots, \lambda_{l}$ are the fundamental dominant weights ([15], p. 67).

We denote by $V(\lambda)$ the finite dimensional irreducible $L$-module with highest weight $\lambda \in \Lambda^{+}$, and denote the corresponding representation by $\pi_{\lambda}$. If

$$
\lambda=\sum_{i=1}^{l} m_{i} \lambda_{l},
$$

we shall of ten label $V(\lambda)$ (or $\left.\pi_{\lambda}\right)$ by $\left(m_{1}, \ldots, m_{l}\right)$. However, we denote the irreducible $s l(2, \mathbf{C})$-modules by $(j)$, where $j=0, \frac{1}{2}, 1, \frac{3}{2}, \ldots$, and $\operatorname{dim}(j)=2 j+1$. This is the conventional notation in physics: in [15] they are denoted by $(2 j)$.

Suppose now that $L^{\prime}$ is a complex semi-simple lie algebra, of rank $l^{\prime}$, such that there is a Lie algebra monomorphism $f: L^{\prime} \rightarrow L$. Then we say that $L^{\prime}$ is embedded in $L$, and call $f$ the embedding. We use primed quantities $H^{\prime}, \Phi^{\prime}, \Delta^{\prime}$, etc. to denote a CSA, root system, base etc. of $L^{\prime} . H^{\prime}$ and $H$ may be chosen such that $f\left(H^{\prime}\right) \subseteq H$ [12], the transpose $f^{*}: H^{*} \rightarrow H^{*}$ will then make sense. Now the $L$-module $V(\lambda)$ may be made into an $L$-module in the obvious way, that is by considering the representation $\pi_{\lambda}$ of of $L^{\prime}$. By Weyl's theorem ([15], p. 28), $V(\lambda)$ is completely reducible:

$$
V(\lambda) \cong \bigoplus_{\lambda^{\prime} \in \Lambda^{\prime}+} n\left(\lambda^{\prime}\right) V^{\prime}\left(\lambda^{\prime}\right) \quad \text { (as an } L^{\prime} \text {-module), }
$$

where $V^{\prime}\left(\lambda^{\prime}\right)$ denotes the irreducible $L^{\prime}$-module with highest weight $\lambda^{\prime} \in \Lambda^{\prime+}$. This decomposition is the branching rule for the representation $\pi_{\lambda}$ restricted to $L^{\prime}$. Clearly, if $\mu \in H^{*}$ is a weight of $H$ on $V(\lambda)$, then $f^{*}(\mu) \in H^{\prime *}$ is a weight of $H^{\prime}$ on $V(\lambda)[12]$.

We say that two embeddings $f_{1}, f_{2}$ of $L^{\prime}$ in $L$ are equivalent (written $f_{1} \sim f_{2}$ ) if they always give rise to the same branching rules for representations of $L$, that is for every $\lambda \in \Lambda^{+}$, the two $L^{\prime}$-module structures induced on $V(\lambda)$ by $f_{1}, f_{2}$ are isomorphic. It turns out $[12,14]$, that an embedding $f: L^{\prime} \rightarrow L$ can be specified up to equivalence by giving the branching rule for just one irreducible $L$-module $V(\omega)$ in the following cases:

\begin{tabular}{cc}
\hline$L$ & $\omega$ \\
\hline$A_{1}$ & $\lambda_{1}$ \\
$B_{1}$ & $\lambda_{1}$ \\
$C_{1}$ & $\lambda_{1}$ \\
$G_{2}$ & $\lambda_{1}$ \\
$F_{4}$ & $\lambda_{4}$ \\
$E_{6}$ & $\lambda_{1}$ \\
\hline
\end{tabular}


For the remaining simple algebras, however, we need to give the branching rules for two irreducible $L$-modules $V(\omega), V\left(\omega^{\prime}\right)$ :

\begin{tabular}{lll}
\hline$L$ & $\omega$ & $\omega^{\prime}$ \\
\hline$D_{1}$ & $\lambda_{1}$ & $\lambda_{1-1}$ \\
$E_{7}$ & $\lambda_{1}$ & $\lambda_{7}$ \\
$E_{8}$ & $\lambda_{1}$ & $\lambda_{8}$ \\
\hline
\end{tabular}

We have numbered the simple roots as in $[15, \mathrm{p} .58]$.

Thus we see that to specify embeddings in the algebras $B_{l}$, and $C_{l}$, we only need give the branching rule for the natural representations, of dimension $2 l+1$ and $2 l$, respectively. However, for $D_{l}$ we must give the branching rules for the natural $2 l$-dimensional representation and for one of the $2^{l-1}$-dimensional spin representations.

Once $f$ has been so specified, we can describe the map $f^{*}$ explicitly $[12,14]$ by computing its action on some basis for $H^{*}$ in terms of a basis for $H^{\prime *}$. It is then straightforward to determine the branching rule for any irreducible $L$-module $V(\lambda)$. To do this, we find the system of weights $\Pi(\lambda)$ of $V(\lambda)$, and then apply $f^{*}$ to obtain the full set of weights for $V(\lambda)$ regarded as an $L^{\prime}$-module. Systematic extraction of the irreducible $L^{\prime}$-modules gives us the required branching rule. We shall use this method in Section 5.

The generalization to the case where $L$ is semi-simple is immediate: we have $L \cong L_{1} \oplus \ldots \oplus L_{r}$, where the $L_{i}$ are ideals of $L$ which are simple Lie algebras of rank $l_{i}$. The irreducible $L$-modules are of the form $V\left(\lambda^{(1)}\right) \otimes \ldots \otimes V\left(\lambda^{(k)}\right)$, where $\left.V \lambda^{(i)}\right)$ is an irreducible $L_{i}$-module $(1 \leqslant i \leqslant r)$. The notation $\left(m_{1}, \ldots, m_{1}\right)$ for the irreducible representations of a simple Lie algebra can be generalized to $\left(\mathrm{m}_{1}^{(1)}, \ldots, m_{l_{1}}^{(1)} ; \ldots\right.$; $\left.m_{1}^{(r)}, \ldots, m_{l_{r}}^{(r)}\right)$ for the irreducible representations of $L$. Again, we make an exception for $\operatorname{so}(4, \mathrm{C}) \cong s l(2, \mathrm{C}) \oplus \operatorname{sl}(2, \mathrm{C})$, and use $(k, l)$, not $(2 k ; 2 l)$, to denote its irreducible representations. Thus, for example, the four-vector representation is $\left(\frac{1}{2}, \frac{1}{2}\right)$, and the adjoint representation is $(1,0) \oplus(0,1)$.

This method of finding branching rules is completely general, and conceptually is the most appealing one. However, it is often cumbersome to apply, because we have to know the set $\Pi(\lambda)$ of weights of $V(\lambda)$. Finding the multiplicity of a weight is quite difficult, especially when the rank of $L$ or the dimension of $V(\lambda)$ is large. So wherever possible, we use branching rules obtained by classical tensor and spinor methods: these rules are only available for the so-called "natural" embedding, for example $s o(n) \subset s o(n+1), s p(n) \oplus s p(m) \subset s p(n+m)$, and so on. We shall not describe these rules in detail (see King [16]).

We remark, in connection with this, that Bauer only considered natural embeddings of $s o(4, C) \subset L$, and consequently missed many more exotic possibilities (see [9], p. 127). 
Now we consider the question of the invariant description of a "vector operator" such as $\left\{\alpha^{\mu}\right\}$, following some unpublished notes by Hannabuss. If $L$ is a complex semi-simple Lie algebra, $\left(\pi_{\lambda}, V(\lambda)\right)$ is some finite dimensional irreducible representation of $L$, and $(\pi, V)$ is any finite dimensional representation of $L$, then we call the vector space

$$
\mathscr{T}=\operatorname{Hom}_{L}(V(\lambda) \otimes V, V)
$$

the space of tensor operators for the pair $(V(\lambda), V)$. By using the canonical isomorphism $V^{*} \otimes W \cong \operatorname{Hom}(V, W)$, we see that

$$
\mathscr{T} \cong \mathscr{T}^{\prime}=\operatorname{Hom}_{L}(V(\lambda), \quad \text { End } V)
$$

This enables us to identify $\mathscr{T}$ with $\mathscr{T}^{\prime}$. We recall that the $L$-module action on End $V$ is given by

$$
x . A=[\pi(x), A]=\pi(x) A-A \pi(x) \quad(x \in L, A \in \text { End } V) .
$$

Choose now $T \in \mathscr{T}(T \neq 0)$, and let $\left\{w_{i} \mid 1 \leqslant i \leqslant m\right\}$ be a basis for $V(\lambda)$. Define $T_{i} \in$ End $V, 1 \leqslant i \leqslant m$, by

$$
T_{i}(v)=T\left(w_{i} \otimes v\right), \quad(v \in V) .
$$

Then it is clear that, if $L$ acts on $\mathrm{V}(\lambda)$ by

$$
x . w_{l}=\sum_{j=1}^{m} c_{j i} w_{j}, \quad\left(x \in L, c_{j i} \in \mathbf{C}\right),
$$

it follows that

$$
x . T_{i}=\sum_{j=1}^{m} c_{j i} T_{j}
$$

This is the usual conception of a tensor operator: a set of operators $\left\{T_{i}\right\}$ which "transform like" some representation of $L$ under commutation.

Finally, we observe that if $\Pi(\lambda)=\left\{v_{1}, \ldots, v_{m}\right\}$ is the set of weights of $V(\lambda)$, and we choose $w_{l}$ to be in the weight space $V(\lambda)_{v i}(1 \leqslant i \leqslant m)$, where

$$
V(\lambda)_{v_{i}}=\left\{w \in V(\lambda) \mid h \cdot w=v_{i}(h) w \text { for all } h \in H\right\},
$$

then the operator $T_{i}$ is a "shift operator" on $V$ in the sense that

$$
T_{i}: V_{\mu} \rightarrow V_{\mu+v_{i}}
$$

\section{Classification of wave equations}

In this section we describe the structure, of wave equations of the form (1.1). As stated in the Introduction, we essentially must list all the minimal complex Lie algebras $L \supset \operatorname{so}(4, \mathrm{C})$ such that the adjoint representation ad $L$ of $L$ contains a copy of the vector representation $\left(\frac{1}{2}, \frac{1}{2}\right)$. Stated in this "analytic" form, the problem is very difficult to attack: we shall use a "synthetic" procedure.

To do this, we use the results of Section 2 on tensor operators, specializing to 
the case $L=s o(4, \mathrm{C})$ and $V(\lambda)=\left(\frac{1}{2}, \frac{1}{2}\right)$. We write

$$
(\pi, V)=\left(\bigoplus_{j=1}^{t} \pi_{j}, \bigoplus_{j=1}^{t} V_{j}\right)
$$

where $\pi_{j}$ denotes the irreducible representation $\left(k_{j}, l_{j}\right)$ of $s o(4, \mathbf{C})$. An irreducible representation $(k, l)$ may occur more than once in $V$. It is clear that there will exist a non-zero four-vector operator $\left\{T_{i} \mid 1 \leqslant i \leqslant 4\right\}$ on $V$ if, and only if, the space $\mathscr{T}=\mathrm{Hom}_{\text {so(4, } \mathrm{C})}\left(\left(\frac{1}{2}, \frac{1}{2}\right) \otimes V, V\right)$ of vector operators is non-trivial. In other words, $V$ must occur as an $s o(4, \mathrm{C})$-sub-module of $\left(\frac{1}{2}, \frac{1}{2}\right) \otimes V$; or, equivalently, $\left(\frac{1}{2}, \frac{1}{2}\right)$ must occur as an $s o(4, \mathrm{C})$-sub-module of End $V \cong V^{*} \otimes V$. We can now easily derive the following result:

Proposition 3.1. Let $V$ be as above, and let p be the number of distinct pairs $\left(V_{r}, V_{s}\right)$ of linked irreducible so $(4, \mathrm{C})$-sub-modules of $V$, where we say $V_{r}$ is linked to $V_{s}$ if $k_{s}=k_{r} \pm \frac{1}{2}$ and (independently) $l_{s}=l_{r} \pm \frac{1}{2}$. Then $\operatorname{dim} \mathscr{T}=2 p$.

Proof. Every representation of $s o(4, \mathrm{C})$ is equivalent to its contragredient [17], and so

$$
\text { End } V \cong V^{*} \otimes V \cong V \otimes V \cong \underset{1 \leqslant r, s \leqslant 1}{\bigoplus}\left[\left(k_{r}, l_{r}\right) \otimes\left(k_{s}, l_{s}\right)\right]
$$

But each term in this sum can be decomposed using the well-known rule

$$
\left(k_{r}, l_{r}\right) \otimes\left(k_{s}, l_{s}\right) \cong \bigoplus_{k=\left|k_{r}-k_{s}\right|}^{k_{r}+k_{s}} \bigoplus_{l=\left|l_{r}-l_{s}\right|}^{l_{r}+l_{s}}(k, l) .
$$

Clearly, $\left(\frac{1}{2}, \frac{1}{2}\right)$ occurs in $\left(k_{r}, l_{r}\right) \otimes\left(k_{s}, l_{s}\right)$ exactly when $k_{s}=k_{r} \pm \frac{1}{2}, l_{s}=l_{r} \pm \frac{1}{2}$. The result now follows.

We see that there are two kinds of linkage; either $k_{r}+l_{r}=k_{s}+l_{s}$ or $k_{r}+l_{r}=k_{s}+l_{s} \pm 1$. They were called by Bhabha Type I and Type II, respectively.

The result gives us the number of linearly independent vector operators in End $V$. Given any vector operator $\left\{T_{i}\right\}$, we let $S$ be the Lie sub-algebra of $s l(V)$ generated by $\left\{\pi(x), T_{i} \mid x \in \operatorname{so}(4, \mathrm{C}), 1 \leqslant i \leqslant 4\right\}$. Clearly, we can restrict our attention to those $V$ which are indecomposable as $S$-modules, since any other $V$ is constructible from these. Now if $T \in \mathscr{T}, T$ has an obvious decomposition as $\sum_{1 \leqslant r, s \leqslant 1} T_{r s}$, where $T_{r s} \in$ Hom $_{s o(4, \mathrm{C})}\left(V(\lambda) \otimes V_{s}, V_{r}\right)$. The "components" $T_{1}$ of $T$ have a corresponding decomposition into matrix blocks, which we write as $\left(r\left|T_{i}\right| s\right)$, or, following Bhabha [3], as $\left(k_{r}, l_{r}\left|T_{i}\right| k_{s}, l_{s}\right)$. If $\left(r\left|T_{i}\right| s\right) \neq 0$, we write $V_{r} \leftarrow V_{s}$, and we call this a one-way coupling of $V_{s}$ to $V_{r}$. This is obviously only possible when $V_{r}$ is linked to $V_{s}$.

It is useful to describe the situation graphically. The point $(k, l)$ in the plane is taken to represent the irreducible $s o(4, \mathrm{C})$-module $(k, l)$. If $V_{r} \leftarrow V_{s}$, we draw a directed line from $\left(k_{s}, l_{s}\right)$ to $\left(k_{r}, l_{r}\right)$. We see now that an indecomposable $V$ has a 
graph consisting of a set of points in the plane, with various one- and two-way couplings, which cannot be written as the sum of two mutually uncoupled subgraphs.

In this paper we shall limit ourselves to the case where $S$ acts irreducibly on $V$. Since it consists of trace zero matrices, $S$ will then be semi-simple. As pointed out by Wightman [18], there is no general argument which shows that all wave equations with $\kappa \neq 0$ must be of this type, although it is easy to see that a wave equation of the type $V_{1} \rightarrow V_{2}$ is inconsistent with $\kappa \neq 0$. If $S$ acts reducibly on $V$, then $S$ is not semi-simple; the representations of such algebras are not very well known.

It is clear that the graph of an irreducible $V$ will be such that any two points in the graph are coupled by a suitable directed path. If the graph has no closed loops, then all the couplings must be two-way; that is $V_{1} \rightleftharpoons V_{2} \rightleftharpoons \ldots \rightleftharpoons V_{r}$. But if closed loops are present, there can be one-way couplings. For example, if $V_{1}=\left(0, \frac{1}{2}\right), V_{2}=\left(\frac{1}{2}, 1\right), V_{3}=\left(1, \frac{1}{2}\right)$ and $V_{4}=\left(\frac{1}{2}, 0\right)$, then $V_{1} \rightarrow V_{2} \rightarrow V_{3} \rightarrow V_{4} \rightarrow V_{1}$ corresponds to an irreducible $V$. We shall consider this example later on.

Let us revert to the structure of $V$ as an $s o(4, \mathrm{C})$-module. It is well known that the irreducible representation $\left(\pi_{r}, V_{r}\right)$ of $s o(4, \mathrm{C})$ is symplectic if $k_{r}+l_{r}$ is halfintegral, and orthogonal if $k_{r}+l_{r}$ is integral [17]. This means that there exists a non-degenerate bilinear form $b_{r}\left(v, v^{\prime}\right)$ on $V_{r}$, which is antisymmetric (symmetric) according as $k_{r}+l_{r}$ is half-integral (integral), such that

$$
b_{r}\left(x \cdot v, v^{\prime}\right)=-b_{r}\left(v, x \cdot v^{\prime}\right) \text { for all } x \in \operatorname{so}(4, \mathrm{C}) .
$$

Clearly, if $V_{r}$ is linked to $V_{s}$, then $V_{r}$ and $V_{s}$ are either both symplectic or both orthogonal. Thus, an irreducible $S$-module $V$ will be either symplectic or orthogonal as an $s o(4, \mathrm{C})$-module. In fact, we have the embedding

$$
s o(4, \mathrm{C}) \subseteq \bigoplus_{r=1}^{\ell} s p\left(V_{r}\right) \subseteq s p(V)
$$

or

$$
s o(4, \mathbf{C}) \subseteq \bigoplus_{r=1}^{t} s o\left(V_{r}\right) \subseteq s o(V),
$$

where $s p(V)(s o(V))$ denotes the Lie algebra of linear transformations on $V$ which are skew relative to the antisymmetric (symmetric) form $b$ induced by the $b_{r}(1 \leqslant r \leqslant t)$. These two distinct kinds of irreducible wave equations are of course those describing particles of half-integral and integral spin, respectively. We shall usually be able to treat these cases concurrently.

We note here that if each irreducible $s o(4, \mathrm{C})$-module occurs an even number of times in $V$, then, as well as the embedding $s o(4, \mathrm{C}) \subseteq s p(V)(s o(V))$, we can also have $s o(4, \mathrm{C}) \subseteq s o(V)(s p(V))$. This is because $W \oplus W^{*} \cong W \oplus W$ is both orthogonal and 
symplectic for any so(4, C)-module $W[17]$. However, we shall not pursue this here, but use the chain (3.2), which is applicable for all $s o(4, \mathrm{C})$-modules.

Since we have an embedding of $s o(4, \mathrm{C})$ in $s p(V)(s o(V))$, it is natural to ask whether $S$ is contained in the same algebra. Now each form $b_{r}$ on $V_{r}$ is only determined up to a non-zero scalar multiple $\delta_{r}$ and $s p\left(V_{r}\right)\left(s o\left(V_{r}\right)\right)$ is unaffected by changing $\delta_{r}$. However, $s p(V)(s o(V))$ depends sharply on the choice of the $\delta_{r}$. So we should really ask if, given some vector operator $\left\{T_{i}\right\}$, there is a choice of constants $\delta_{r}$ such that $\left\{T_{i}\right\} \subseteq s p(V)(s o(V))$, and hence $S \subseteq s p(V)(s o(V))$. We answer this question later on, but we can establish here an existence result.

Proposition 3.2. Let $V$, p be as in Proposition 3.1. Choose any values of the constants $\delta_{r}$, thus fixing $s p(V)(s o(V))$. Then there are exactly $p$ linearly independent vector operators $\left\{T_{i}\right\}$ in $s p(V)(s o(V))$.

Proof. (a) Suppose $V$ is symplectic as an so(4,C)-module. We shall use King's results [16] to obtain the branching rule for

$$
s p(V) \rightarrow \bigoplus_{r=1}^{t} s p\left(V_{r}\right) .
$$

To do this, we use the Young diagram notation $\langle\lambda\rangle=\left\langle r_{1}, \ldots, r_{l}\right\rangle$ (with $r_{1}, \ldots, r_{l}$ non-negative integers and $\left.r_{1} \geqslant r_{2} \geqslant \ldots r_{l} \geqslant 0\right)$ as an alternative label for the irreducible $s p(2 l)$-module $V(\lambda)=\left(m_{1}, \ldots, m_{l}\right)$, where

$$
r_{j}=\sum_{i=j}^{l} m_{i}, \quad 1 \leqslant j \leqslant l,
$$

that is, $m_{j}=r_{j}-r_{j+1}(1 \leqslant j \leqslant l-1)$ and $m_{l}=r_{l}$.

In this notation, the branching rule for

is given by

$$
s p(2 r+2 s) \rightarrow s p(2 r) \oplus s p(2 s)
$$

$$
\langle\lambda\rangle \rightarrow \underset{\zeta, \beta}{\bigoplus}\langle(\lambda / \zeta) ;(\zeta / \beta)\rangle,
$$

where the summation is taken over all partitions $\zeta$, and over those $\beta$ which are the conjugate of an even partition [16]. The division refers to the usual division of $S$-functions [19]. In particular, for the adjoint representation $\langle 2\rangle$ of $s p(2 r+2 s)$ we have

$$
\langle 2\rangle \rightarrow\langle 2 ; 0\rangle \oplus\langle 0 ; 2\rangle \oplus\langle 1 ; 1\rangle .
$$

A simple induction argument then yields, for the reduction (3.3):

$$
\langle 2\rangle \rightarrow\langle 2 ; 0 ; \ldots ; 0\rangle \oplus\langle 0 ; 2 ; 0 ; \ldots ; 0\rangle \oplus \ldots \oplus\langle 0 ; \ldots ; 0 ; 2\rangle \oplus \underset{1 \leqslant r<s \leqslant t}{\bigoplus} W_{r s},
$$

where $W_{r s}$ denotes the irreducible $\bigoplus_{r=1}^{t} s p\left(V_{r}\right)$-module

$$
\langle 0 ; \ldots ; 0 ; 1 ; 0 ; \ldots ; 0 ; 1 ; 0 ; \ldots ; 0\rangle
$$


with one in the $r$ and $s$ positions and zeros elsewhere. It is clear that $W_{r s}$ contains the $\operatorname{so}(4, \mathrm{C})$-module $\left(\frac{1}{2}, \frac{1}{2}\right)$ once if, and only if, $V_{r}$ is linked to $V_{s}$, and the result follows.

(b) If $V$ is orthogonal, the proof is similar. In this case the Young diagram notation $[\lambda]=\left[p_{1}, \ldots, p_{l}\right]$ for irreducible $s o(2 l+1)$-modules is related to the highest weight notation $\left(m_{1}, \ldots, m_{l}\right)$ by

$$
\begin{aligned}
& p_{j}=\sum_{i=j}^{l-1} m_{i}+\frac{1}{2} m_{l}, \quad 1 \leqslant j \leqslant l-1, \\
& p_{l}=\frac{1}{2} m_{l},
\end{aligned}
$$

while for $s o(2 l)$ we have

$$
\begin{aligned}
p_{j} & =\sum_{i=j}^{l-2} m_{i}+\frac{1}{2}\left(m_{l-1}+m_{l}\right), \quad 1 \leqslant j \leqslant l-2, \\
p_{l-1} & =\frac{1}{2}\left(m_{l-1}+m_{l}\right) \\
p_{l} & =\frac{1}{2}\left(m_{l}-m_{l-1}\right) .
\end{aligned}
$$

The adjoint representation of $s o(2 l+1)$ or $s o(2 l)$ is $\left[1^{2}\right]$, and we can deduce, from [16]:

$$
\begin{gathered}
s o(V) \rightarrow \bigoplus_{r=1}^{t} \operatorname{so}\left(V_{r}\right), \\
{\left[1^{2}\right] \rightarrow\left[1^{2} ; 0 ; \ldots ; 0\right] \oplus \ldots \oplus\left[0 ; \ldots ; 0 ; 1^{2}\right] \oplus \underset{1 \leqslant r<s \leqslant 1}{\bigoplus} Z_{r s},}
\end{gathered}
$$

with $Z_{r s}$ denoting the irreducible $\bigoplus_{r=1}^{t} s o\left(V_{r}\right)$-module

$$
[0 ; \ldots ; 0 ; 1 ; 0 ; \ldots ; 0 ; 1 ; 0 ; \ldots ; 0]
$$

with one in the $r$ and $s$ positions and zeros elsewhere. As in (a), the result follows.

To carry out calculations, it is necessary to write down explicit expressions for the matrix blocks $\left(r\left|T_{i}\right| s\right)$ of the vector operator. We can do this fairly easily by using the definition of a vector operator given in Section 2.

It is formally convenient to describe the transformation properties of operators in spinor form. Upper case Italic letters will be used for spinor indices: they take the values 1,2 . Thus instead of $\left\{T_{i}\right\}$, we now write $\left\{T^{A B}\right\}$, which is related to the $\alpha^{\mu}$ occurring in (1.1) by [3]:

$$
\left(\begin{array}{cc}
T^{1 \mathrm{i}} & T^{1 \dot{2}} \\
T^{2 \mathrm{i}} & T^{2 \dot{\mathrm{z}}}
\end{array}\right)=\left(\begin{array}{ll}
\alpha^{0}+\alpha^{3} & \alpha^{1}-i \alpha^{2} \\
\alpha^{1}+i \alpha^{2} & \alpha^{0}-\alpha^{3}
\end{array}\right)
$$

Suppose that $\left\{h, x, y, h^{\prime}, x^{\prime}, y^{\prime}\right\}$ is the usual basis for $\operatorname{so}(4, \mathbf{C})=s l(2, \mathbf{C}) \oplus \operatorname{sl}(2, \mathbf{C})$, with 


$$
\begin{aligned}
{[h x] } & =2 x, \quad[h y]=-2 y, \quad[x y]=h, \\
{\left[h^{\prime} x^{\prime}\right] } & =2 x^{\prime}, \quad\left[h^{\prime} y^{\prime}\right]=-2 y^{\prime}, \quad\left[x^{\prime} y^{\prime}\right]=h^{\prime}
\end{aligned}
$$

and primed and unprimed quantities commuting. Then corresponding to the representation $\left(\pi_{r}, V_{r}\right)$ of $s o(4, C)$ we can define two symmetric spinors $K^{A B}\left(k_{r}\right)$, $L^{A B}\left(l_{r}\right)$ by [3]:

$$
\begin{aligned}
& K_{1}{ }^{1}=-K_{2}{ }^{2}=K_{3}, \quad K_{2}{ }^{1}=K_{-}, \quad K_{1}{ }^{2}=K_{+}, \\
& L_{\mathrm{i}}{ }^{\mathrm{i}}=-L_{2}{ }^{\mathrm{i}}=-L_{3}, \quad L_{\dot{2}}^{\mathrm{i}}=-L_{+}, \quad L_{\mathrm{i}}{ }^{\mathrm{i}}=-L_{-},
\end{aligned}
$$

where

$$
\begin{aligned}
& K_{3}=\frac{1}{2} \pi_{r}(h), \quad L_{3}=\frac{1}{2} \pi_{r}\left(h^{\prime}\right), \\
& K_{+}=\pi_{r}(x), \quad L_{+}=\pi_{r}\left(x^{\prime}\right), \\
& K_{-}=\pi_{r}(y), \quad L_{-}=\pi_{r}\left(y^{\prime}\right) .
\end{aligned}
$$

We shall choose the usual basis for $V_{r}$ :

$$
\left\{v_{k_{r}, m_{r} ; i_{r}, n_{r}} \mid-k_{r} \leqslant m_{r} \leqslant k_{r},-l_{r} \leqslant n_{r} \leqslant l_{r}\right\} .
$$

In this basis, the matrix elements of the $K$ 's are

$$
\begin{aligned}
\left(K_{3}\right)_{m_{r} n_{r} ; m_{r^{\prime} n_{r^{\prime}}}}=m_{r} \delta_{m_{r} m_{r}} \delta_{n_{r} n_{r^{\prime}}}, \\
\left(K_{ \pm}\right)_{m_{r} n_{r}: m_{r^{\prime} n_{r^{\prime}}}}=\left[\left(k_{r} \mp m_{r^{\prime}}\right)\left(k_{r} \pm m_{r^{\prime}}+1\right)\right]^{\frac{1}{2}} \delta_{m_{r^{\prime}, m_{r^{\prime}} \pm 1} \delta_{n_{r} n_{r^{\prime}}}}
\end{aligned}
$$

Similar formulae hold for the $L$ 's. Note that the matrix $B_{r}$ of the bi-linear nondegenerate form $b_{r}$ on $V_{r}$, which we discussed earlier, is given by

$$
\left(B_{r}\right)_{m_{r} n_{r}: m_{r} n_{r}}=(-1)^{-\left(m_{r}+n_{r}\right)} \delta_{m_{r}-m_{r}} \cdot \delta_{n_{r},-n_{r}} \text {. }
$$

It is easy to see that $\left(B_{r}^{\mathrm{T}}\right.$ denoting the transpose of $\left.B_{r}\right)$ :

$$
B_{r}^{\mathrm{T}}=(-1)^{2\left(k_{r}+l_{r}\right)} B_{r}
$$

and also that (3.1) is satisfied.

Now $\left\{T^{A B}\right\}$ must transform like the basis $\left\{W_{A B}\right\}$ of $\left(\frac{1}{2}, \frac{1}{2}\right)$, which is related to the canonical basis by

$$
W_{A B}=(-1)^{\frac{1}{2}-\hat{A}} v_{\frac{1}{2},-\hat{A} ; \frac{1}{2}, \hat{B}}
$$

where $\hat{A}, \hat{B}=\frac{1}{2},-\frac{1}{2}$ as $A, B=1,2$. From the definition of $T^{A B}$, we see that

$$
\begin{aligned}
& \left(r\left|T^{A B}\right| s\right): V_{s} \rightarrow V_{r} \\
& \quad v_{k_{s} \cdot m_{s} ; l_{s}, n_{s}} \rightarrow T_{r s}\left(W_{A B} \otimes v_{k_{s}, m_{s} ; i_{s}, n_{s}}\right) .
\end{aligned}
$$

The right-hand side may be written as:

$$
a_{r s}(-1)^{\frac{1}{2}-\hat{A}}\left(k_{s}\right)\left(l_{s}\right) \sum_{m_{r}, n_{s}}\left(\frac{1}{2} k_{s} k_{r} m_{r} \mid-\hat{A} m_{s}\right)\left(\frac{1}{2} l_{s} l_{r} n_{r} \mid \hat{B} n_{s}\right) v_{k_{r}, m_{r}: l_{r}, n_{r}} \quad\left(a_{r s} \in \mathbf{C}\right) .
$$

In (3.6), $\left(j_{1} j_{2} j m \mid m_{1} m_{2}\right)$ denotes the Clebsch-Gordan coefficient for the coupling 
of $j_{1}$ and $j_{2}$ to give $j_{3}$, and $(k)$ means $(2 k+1)^{\frac{1}{2}}$. Thus we see that the block $\left(r\left|T^{A B}\right| s\right)$ has matrix elements

$$
\left(r\left|T^{A B}\right| s\right)_{m_{r}, n_{r} ; m_{s}, n_{s}}=a_{r s}(-1)^{\frac{1}{2}-\bar{A}}\left(k_{s}\right)\left(l_{s}\right)\left(\frac{1}{2} k_{s} k_{r} m_{r} \mid-\hat{A} m_{s}\right)\left(\frac{1}{2} l_{s} l_{r} n_{r} \mid \hat{B} n_{s}\right) .
$$

We shall also find it convenient to express the blocks $\left(r\left|T^{A B}\right| s\right)$ in terms of the spinor matrices $u^{A}(k), v^{A}(k), u^{B}(l), v^{B}(l)$. For Type I coupling $\left(k_{s}=k_{r}+\frac{1}{2}, l_{s}=l_{r}-\frac{1}{2}\right)$ we have:

$$
\begin{aligned}
& \left(r\left|T^{A B}\right| s\right)=a_{r s} v^{A}\left(k_{r}+\frac{1}{2}\right) \otimes u^{B}\left(I_{r}\right), \\
& \left(s\left|T^{A B}\right| r\right)=a_{s r} u^{A}\left(k_{r}+\frac{1}{2}\right) \otimes v^{B}\left(I_{r}\right),
\end{aligned}
$$

while for Type II coupling $\left(k_{s}=k_{r}+\frac{1}{2}, l_{s}=l_{r}+\frac{1}{2}\right)$,

$$
\begin{aligned}
& \left(r\left|T^{A B}\right| s\right)=a_{r s} v^{A}\left(k_{r}+\frac{1}{2}\right) \otimes v^{B}\left(l_{r}+\frac{1}{2}\right), \\
& \left(s\left|T^{A \dot{B}}\right| r\right)=a_{s r} u^{A}\left(k_{r}+\frac{1}{2}\right) \otimes u^{\dot{B}}\left(l_{r}+\frac{1}{2}\right) .
\end{aligned}
$$

The spinor matrices are given by

$$
\begin{aligned}
u^{A}\left(k+\frac{1}{2}\right)_{m m^{\prime}} & =(-1)^{\frac{1}{2}-\hat{A}}(k)\left(\frac{1}{2} k k+\frac{1}{2} m \mid-\hat{A} m^{\prime}\right), \\
v^{A}(k)_{m m^{\prime}} & =(-1)^{\frac{1}{2}-\hat{A}}(k)\left(\frac{1}{2} k k-\frac{1}{2} m \mid-\hat{A} m^{\prime}\right), \\
u^{B}\left(l+\frac{1}{2}\right)_{m n^{\prime}} & =(l)\left(\frac{1}{2} l l+\frac{1}{2} n \mid n \hat{B}^{\prime}\right), \\
v^{B}(l)_{n n^{\prime}} & =(l)\left(\frac{1}{2} l l-\frac{1}{2} n \mid \hat{B} n^{\prime}\right) .
\end{aligned}
$$

They satisfy the relations

$$
\begin{gathered}
u_{A}\left(k+\frac{1}{2}\right) v^{A}\left(k+\frac{1}{2}\right)=-v_{A}(k) u^{A}(k)=2 k+1, \\
v_{A}(k) v^{A}\left(k+\frac{1}{2}\right)=u_{A}\left(k+\frac{1}{2}\right) u^{A}(k)=0, \\
v^{A}\left(k+\frac{1}{2}\right) u^{B}\left(k+\frac{1}{2}\right)=K^{A B}(k)+(k+1) \varepsilon^{A B}, \\
u^{A}(k) v^{B}(k)=K^{A B}(k)-k \varepsilon^{A B} .
\end{gathered}
$$

Similar relations hold for $u^{B}(l), v^{B}(l)$, if we replace $K$ by $L$. Apart from a change of phase of $v^{A}, v^{B}$, these relations are essentially those given by Bhabha [3].

For each linkage $\left(V_{r}, V_{s}\right)$, the constants $a_{r s}, a_{s r}$ are quite arbitrary: this gives us the $2 p$ linearly independent vector operators mentioned in Proposition 3.1. If we fix these constants, we get a vector operator which can be denoted $T^{A B}(a)$. Now two such vector operators $T^{A B}(a), T^{A B}\left(a^{\prime}\right)$ may be physically equivalent: this is so exactly when they lead to the same mass spectrum, which means that

$$
\begin{gathered}
\alpha^{0}\left(a^{\prime}\right)=Q \alpha^{0}(a) Q^{-1}, \\
{[Q, \pi(x)]=0 \quad \text { for all } x \in \operatorname{so}(4, \mathrm{C}) .}
\end{gathered}
$$


It follows from (3.12) that

and thus

$$
\alpha^{\mu}\left(a^{\prime}\right)=Q \alpha^{\mu}(a) Q^{-1}
$$

$$
T^{A B}\left(a^{\prime}\right)=Q T^{A B}(a) Q^{-1} .
$$

We shall write $T^{A B}\left(a^{\prime}\right) \sim T^{A B}(a)$ if such a $Q$ exists. It is clear that $\sim$ is an equivalence relation; we denote the equivalence class of $T^{A \hat{B}}(a)$ modulo $\sim$ by $\left[T^{A \dot{B}}(a)\right]$.

In order to describe these classes explicitly, we recall that $\pi$ may contain repeated representations. Without loss of generality, we may group together the equivalent subrepresentations of $\pi$, and write

$$
\pi=\bigoplus_{r=1}^{k} n_{r} \varphi_{r},
$$

where $\sum_{r=1}^{k} n_{r}=t, \varphi_{r}$ is $\left(k_{r}, l_{r}\right)$, and $\varphi_{r}, \varphi_{s}$ are in equivalent if $r \neq s . V$ will have a corresponding decomposition

$$
V=\bigoplus_{r=1}^{k} n_{r} W_{r} \equiv \bigoplus_{r=1}^{k} Y_{r}
$$

If $X \in$ End $V$, it can be split up into super-matrix blocks which we denote by $[r|X| s] \in \operatorname{Hom}\left(Y_{s}, Y_{r}\right)$. In particular $T^{A B}$ splits up into blocks $\left[r\left|T^{A B}\right| s\right]$; each of which consists of smaller blocks of the form (3.8) or (3.9) This allows us to make an obvious generalization of (3.8) and (3.9) by writing

$$
\left[r\left|T^{A B}\right| s\right]=A_{r s} \otimes\left(v^{A}\left(k_{r}+\frac{1}{2}\right) \otimes u^{B}\left(l_{r}\right)\right)
$$

and so on, where $A_{r s}$ is now an arbitrary coupling matrix, with $m_{r}$ rows and $m_{s}$ columns. The vector operator may also be denoted by $T^{A B}(A)$.

The most general matrix $Q \in G L(V)$ which commutes with $\pi(s o(4))$ is [6a]:

$$
Q=\bigoplus_{r=1}^{k}\left(Q_{r} \otimes I_{d_{r}}\right),
$$

where $Q_{r} \in G L\left(n_{r}\right)$, and $d_{r}=\operatorname{dim} \varphi_{r}$. Rewriting (3.13) as

we find that

$$
T^{A B}\left(A^{\prime}\right)=Q T^{A B}(A) Q^{-1}
$$

$$
A_{r s}^{\prime}=Q_{r} A_{r s} Q_{s}^{-1} \text {. }
$$

If the graph of $V$ contains a closed loop $\gamma$ of the form

$$
V_{r_{1}} \rightleftharpoons V_{r_{2}} \rightleftharpoons \ldots \rightleftharpoons V_{r n} \leftrightharpoons V_{r_{1}}, \quad n \geqslant 2
$$

(where repeated representations may occur), we define

$$
\begin{aligned}
& a^{+}(\gamma)=a_{r_{1} r_{2}} a_{r_{2} r_{3}} \ldots a_{r_{n-1} r_{n}} a_{r_{n} r_{1}}, \\
& a^{-}(\gamma)=a_{r_{1} r_{n}} a_{r_{n} r_{n-1}} \ldots a_{r_{2} r_{1}} .
\end{aligned}
$$


These cyclic quantities will be very important in the sequel. Once more, we may generalize this notation to apply to a closed "loop" $\Gamma$ of the form

For $1 \leqslant i \leqslant n$, we write

$$
Y_{r_{1}} \rightleftharpoons Y_{r_{2}} \rightleftharpoons \ldots \rightleftharpoons Y_{r_{n}} \rightleftharpoons Y_{r_{1}}
$$

$$
\begin{aligned}
& A^{+}\left(\Gamma, r_{i}\right)=A_{r_{i} r_{i+1}} A_{r_{i+1} r_{i+2}} \ldots A_{r_{n-1} r_{n}} A_{r_{n} r_{1}} \ldots A_{r_{i-1} r_{i}}, \\
& A^{-}\left(\Gamma, r_{i}\right)=A_{r_{i} r_{i-1}} A_{r_{i-1} r_{i-2}} \ldots A_{r_{1} r_{n}} A_{r_{n} r_{n-1}} \ldots A_{r_{i+1} r_{i}} .
\end{aligned}
$$

It is clear from (3.16) that

if and only if

$$
T^{A B}\left(A^{\prime}\right)=Q T^{A B}(A) Q^{-1}
$$

$$
A^{ \pm \prime}\left(\Gamma, r_{i}\right)=Q_{r_{i}} A^{ \pm}\left(\Gamma, r_{i}\right) Q_{r_{i}}^{-1} \text { for every } \Gamma \text {. }
$$

Thus, the class $\left[T^{A B}(A)\right]$ is parameterized by the eigenvalues of $A^{ \pm}\left(\Gamma, r_{i}\right)$, for $\Gamma$ of the form (3.19), and $1 \leqslant i \leqslant n$.

These results are fairly complicated. However, they simplify considerably if we restrict ourselves to the case where $\pi$ has no repeated sub-representations. Reasonable wave equations are usually of this type. If we replace $A$ by $a, \Gamma$ by $\gamma$ : and note that $Q$ is now

$$
\bigoplus_{r=1}^{t} q_{r} I_{d_{r}} \quad\left(q_{r} \in \mathbf{C}\right)
$$

then (3.16) becomes

$$
a_{r s}^{\prime}=q_{r} q_{s}^{-1} a_{r s}
$$

and we see that $(3.21)$ becomes

$$
T^{A B}\left(a^{\prime}\right)=Q T^{A B}(a) Q^{-1}
$$

if and only if

$$
a^{ \pm \prime}(\gamma)=a^{ \pm}(\gamma) \text { for every closed loop } \gamma
$$

In particular, by taking $\gamma$ to be any trivial loop $V_{r} \rightleftharpoons V_{s}$, we have $a_{r s}^{\prime} a_{s r}^{\prime}=a_{r s} a_{s r}$. Thus the equivalence class $\left[T^{A B}(a)\right]$ is parameterized by the $n=p+c$ quantities $a_{r s} a_{s r}$, and $a^{+}(\gamma)$, where $\gamma$ goes over the $c$ non-trivial closed loops in the graph of $V$ : in other words, physically inequivalent vector operators are in one-to-one correspondence with points in $\mathbf{C}^{n}$.

Clearly, the above arguments are still valid if there are some one-way couplings; except that fewer parameters will be needed.

Returning to the general case, we can now answer the question raised earlier; given $T^{A B}(a)$, can we choose the constants $\delta_{r}$ such that $T^{A B}(a)$ is skew relative to the bilinear form with matrix

$$
B=\bigoplus_{r=1}^{\ell} \delta_{r} B_{r}
$$




$$
B T^{A B}+\left(T^{A B}\right)^{\top} B=0 .
$$

The answer is given by the following

TheOREM 3.3. The vector operator $T^{A B}(a)$ is skew with respect to $B=\bigoplus_{r=1}^{t} \delta_{r} B_{r}$ if, and only if, all the couplings are two-way, and $a^{+}(\gamma)=a^{-}(\gamma)$ for every non-trivial closed loop $\gamma$ in the graph of $V$. The constants $\delta_{r}$ are given by:

$$
\delta_{1}=1, \quad \delta_{s}=\frac{a_{r s}}{a_{s r}} \delta_{r} \text { if } V_{r} \rightleftharpoons V_{s} .
$$

In particular, if there are no such loops, so that the graph is an open chain, then $T^{A B}(a)$ is always skew relative to $B$. If $\pi$ has no repeated representations, then the members of the class $\left[T^{A B}(a)\right]$ are either all skew relative to some $B$ or else none of them are.

Proof. We can write (3.23) in the block form

$$
\delta_{r} B_{r}\left(r\left|T^{A B}\right| s\right)+\left(s\left|T^{A B}\right| r\right)^{\mathrm{T}} \delta_{s} B_{s}=0 .
$$

By substituting (3.5) and (3.7) into this equation we obtain

$$
\begin{aligned}
(-1)^{-\left(m_{r}+n_{r}\right)} & \delta_{r} a_{r s}\left(k_{s}\right)\left(l_{s}\right)\left(\frac{1}{2} k_{s} k_{r}-m_{r} \mid-\hat{A} m_{s}\right)\left(\frac{1}{2} l_{s} l_{r}-n_{r} \mid \hat{B} n_{s}\right) \\
& =-\delta_{s} a_{s r}(-1)^{m_{s}+n_{s}}\left(k_{r}\right)\left(l_{r}\right)\left(\frac{1}{2} k_{r} k_{s}-m_{s} \mid-\hat{A} m_{r}\right)\left(\frac{1}{2} l_{r} l_{s}-n_{s} \mid \hat{B} n_{r}\right) .
\end{aligned}
$$

Thus $a_{r s}=0$ if and only if $a_{s r}=0$, so all couplings must be two-way. Using the relation $([20$, p. 10$)$

$$
\left(j_{1} j_{2} j m \mid m_{1} m_{2}\right)=(-1)^{j_{1}-m_{1}} \frac{(j)}{\left(j_{2}\right)}\left(j_{1} j j_{2}-m_{2} \mid m_{1}-m\right),
$$

we get, after some manipulation,

$$
\delta_{s}=\frac{a_{r s}}{a_{s r}} \delta_{r}
$$

as required. If $\gamma$ is the closed loop (3.17), then it is clear that the $\delta_{r}$ are consistently defined exactly when $a^{+}(\gamma)=a^{-}(\gamma)$. Finally if $\pi$ has no repeated representations and $T^{A \hat{B}}\left(a^{\prime}\right) \in\left[T^{A B}(a)\right]$, then $a^{ \pm \prime}(\gamma)=a^{ \pm}(\gamma)$, and so

$$
a^{+}(\gamma)=a^{-}(\gamma) \text { if and only if } a^{+\prime}(\gamma)=a^{-1}(\gamma) .
$$

This completes the proof.

The calculation of the Lie algebra $S(a)$, generated by $T^{A B}(a)$ and $\pi(x)(x \in \operatorname{so}(4, \mathrm{C}))$, is in general extremely complicated. Of course, if $T^{A B}\left(a^{\prime}\right) \in\left[T^{A B}(a)\right]$, then $S\left(a^{\prime}\right)$ is conjugate to $S(a)$, but if $\left[T^{A B}(a)\right]$ and $\left[T^{A B}\left(a^{\prime}\right)\right]$ are disjoint classes, then $S(a)$ may not even be isomorphic to $S\left(a^{\prime}\right)$. To see what can happen, we shall consider some special examples of (irreducible) wave equations. 
The simplest possibility is $t=2$, so that $(\pi, V)=\left(\pi_{1} \oplus \pi_{2}, V_{1} \oplus V_{2}\right)$, where $\pi_{1}=\left(k_{1}, l_{1}\right)$ and $\pi_{2}=\left(k_{2}, l_{2}\right)$. From above, we have $S(a) \subseteq s p(V)(s o(V))$, for any vector operator $T^{A B}(a)$. In this case we have the following

THEOREM 3.4. Suppose the graph of $V$ does not meet the $k$ or $l$ axes. Then $S(a)=\operatorname{sp}(V)(\operatorname{so}(V))$, for any choice of coupling constants $a_{12}, a_{21} \neq 0$.

Proof. (I) If the linkage is Type I, we take $k_{2}=k_{1}+\frac{1}{2}, l_{2}=l_{1}-\frac{1}{2}$, where by hypothesis $k_{1}>0, l_{1}>\frac{1}{2}$. Writing

$$
\begin{aligned}
& 4 R_{A}^{B}=-\left[T^{B C}, T_{C A}\right], \\
& 4 R_{A}^{B}=\left[T_{A C}, T^{C B}\right],
\end{aligned}
$$

we find, using (3.8) and (3.11), that [3]:

$$
\begin{aligned}
& \left(1\left|R_{A}{ }^{B}\right| 1\right)=a_{12} a_{21} l_{1} K_{A}^{B}\left(k_{1}\right), \\
& \left(2\left|R_{A}^{B}\right| 2\right)=-\frac{1}{2} a_{12} a_{21}\left(2 l_{1}+1\right) K_{A}^{B}\left(k_{1}+\frac{1}{2}\right), \\
& \left(1\left|R_{A}^{B}\right| 1\right)=-\frac{1}{2} a_{12} a_{21}\left(2 k_{1}+2\right) L_{A}^{B}\left(l_{1}\right), \\
& \left(2\left|R_{A}^{B}\right| 2\right)=\frac{1}{2} a_{12} a_{21}\left(2 k_{1}+1\right) L_{A}^{B}\left(l_{1}-\frac{1}{2}\right),
\end{aligned}
$$

The off-diagonal blocks of $R_{A}{ }^{B}, R_{A}{ }^{B}$ are of course zero. Now we observe that the coefficients of $K_{A}{ }^{B}\left(k_{1}\right), K_{A}{ }^{B}\left(k_{1}+\frac{1}{2}\right)$ in (3.24) can never be equal; the same is true for the coefficients of $L_{A}^{B}\left(l_{1}\right), L_{A}^{B}\left(l_{1}-\frac{1}{2}\right)$ in (3.25). Also, since $k_{1}>0, l_{1}>\frac{1}{2}$, these matrices are non-zero. Thus the linear span $R$ of the matrices $R_{A}{ }^{B}$ will be of the form

$$
R=\left\{\gamma(x)=\xi_{1} \pi_{1}(x) \oplus \xi_{2} \pi_{2}(x) \mid x \in \operatorname{sl}(2, \mathbf{C})\right\},
$$

with $\xi_{1} \neq \xi_{2}$; similarly the linear span $R^{\prime}$ of the matrices $R_{A}{ }^{B}$ will be of the form

$$
R^{\prime}=\left\{\gamma^{\prime}\left(x^{\prime}\right)=\xi_{1}^{\prime} \pi_{1}\left(x^{\prime}\right) \oplus \xi_{2}^{\prime} \pi_{2}\left(x^{\prime}\right) \mid x^{\prime} \in s l(2, \mathbf{C})^{\prime}\right\},
$$

where $\xi_{1}^{\prime} \neq \xi_{2}^{\prime}$. Since $R, R^{\prime} \subseteq S(a)$, we see that $S(a)$ also contains the matrices

$$
\begin{aligned}
& \Delta_{1}(x)=\pi_{1}(x) \oplus 0=\frac{\xi_{2} \pi(x)-\gamma(x)}{\xi_{2}-\xi_{1}} \text { or } \frac{\xi_{2}^{\prime} \pi(x)-\gamma^{\prime}(x)}{\xi_{2}^{\prime}-\xi_{1}^{\prime}} \\
& \Delta_{2}(x)=0 \oplus \pi_{2}(x)=\frac{\xi_{1} \pi(x)-\gamma(x)}{\xi_{1}-\xi_{2}} \text { or } \frac{\xi_{1}^{\prime} \pi(x)-\gamma^{\prime}(x)}{\xi_{1}^{\prime}-\xi_{2}^{\prime}}
\end{aligned}
$$

according as $x$ lies in $\operatorname{sl}(2, \mathrm{C})$ or $\operatorname{sl}(2, \mathrm{C})^{\prime}$.

We now write, say,

$$
T^{1 \grave{i}}=\left(\begin{array}{ll}
0 & X \\
\tilde{X} & 0
\end{array}\right) \text {, }
$$


where $\tilde{X}$ is the matrix uniquely determined by $X$ from the requirement that $T^{A B}$ be skew relative to $B$. Then if $m, n \in \mathbf{Z}^{+}$and $x, y \in \operatorname{so}(4, \mathbf{C})$ we have

$$
\begin{aligned}
&\left(\operatorname{ad} \Delta_{1}(x)\right)^{m}\left(\operatorname{ad} \Delta_{2}(y)\right)^{n} T^{1 \mathrm{i}} \\
& \quad=\left(\begin{array}{cc}
0 & (-1)^{n}\left[\pi_{1}(x)\right]^{m} X\left[\pi_{2}(y)\right]^{n} \\
(-1)^{n}\left\{\left[\pi_{1}(x)\right]^{m} X\left[\pi_{2}(y)\right]^{n}\right\}^{\sim} & 0
\end{array}\right) .
\end{aligned}
$$

By taking suitable repeated commutators, we find that $S(a)$ contains all the matrices of the form

$$
\left(\begin{array}{cc}
0 & \pi_{1}(u) X \pi_{2}\left(u^{\prime}\right) \\
{\left[\pi_{1}(u) X \pi_{2}\left(u^{\prime}\right)\right]^{\sim}} & 0
\end{array}\right), u, u^{\prime} \in U \backslash\{1\} .
$$

Here $U$ denotes the universal enveloping algebra of $s o(4, \mathbf{C})$, and 1 its identity.

Now $\pi_{i}(U \backslash\{1\})$, for $i=1,2$, is an irreducible algebra of linear transformations in a finite dimensional vector space, and so it must be the complete matrix algebra End $V_{l}$. Thus $S(a)$ contains all the matrices

$$
\left(\begin{array}{cc}
0 & A_{1} X A_{2} \\
{\left[A_{1} X A_{2}\right]^{\sim}} & 0
\end{array}\right), \quad A_{i} \in \text { End } V_{i} .
$$

If $X$ has matrix elements $\left\{\xi_{p q}\right\}$, with $\xi_{k l} \neq 0$ (say), and we choose $A_{1}=e_{k^{\prime} k}, A_{2}=e_{l l^{\prime}}$ (where $e_{i j}$ denotes the matrix with 1 at the intersection of the $i$ th row and the $j t h$ column, and zeros elsewhere), then clearly

$$
A_{1} X A_{2}=\xi_{k l} e_{k^{\prime} l} \cdot
$$

By taking suitable linear combinations of such matrices, it is clear that the matrices

$$
\left(\begin{array}{ll}
0 & A \\
\tilde{A} & 0
\end{array}\right) \quad(A \text { arbitrary })
$$

belong to $S(a)$. These matrices generate all of $s p(v)(s o(V))$, and so

$$
S(a)=s p(V)(s o(V)) \text {. }
$$

(II) If the linkage is type II, we take $k_{2}=k_{1}+\frac{1}{2}, l_{2}=l_{1}+\frac{1}{2}$, where $k_{1}, l_{1}>0$. We find that

$$
\begin{aligned}
& \left(1\left|R_{A}{ }^{B}\right| 1\right)=-\frac{1}{2} a_{12} a_{21}\left(2 l_{1}+2\right) K_{A}^{B}\left(k_{1}\right), \\
& \left(2\left|R_{A}{ }^{B}\right| 2\right)=\frac{1}{2} a_{12} a_{21}\left(2 l_{1}+1\right) K_{A}^{B}\left(k_{1}+\frac{1}{2}\right), \\
& \left(1\left|R_{A}{ }^{B}\right| 1\right)=-\frac{1}{2} a_{12} a_{21}\left(2 k_{1}+2\right) L_{A}^{B}\left(l_{1}\right), \\
& \left(2\left|R_{A}{ }^{B}\right| 2\right)=\frac{1}{2} a_{12} a_{21}\left(2 k_{1}+1\right) L_{A}^{B}\left(l_{1}+\frac{1}{2}\right) .
\end{aligned}
$$

The arguments used in (I) now apply word for word, and $S(a)$ must be all of $s p(V)(s o(V))$. 
We observe that Theorem 3.4 provides us with a large class of wave equations for which $S(a)$ is not isomorphic to $s o(5)$, contrary to the claims made by Bauer [9] and Lorente, Huddleston and Roman [10]. The crucial point is to realize that, although the diagonal blocks of $R_{A}{ }^{B}, R_{A}{ }^{B}$ are multiples of $K_{A}{ }^{B}, L_{A}{ }^{B}$, respectively, these constants are different for each block: thus $\operatorname{dim} S(a)>\operatorname{dim} s o(5)$. Note that we excluded the cases (I) $k_{1}=0, l_{1}=\frac{1}{2}$, and (II) $k_{1}=l_{1}=0$. For these equations, $R_{A}{ }^{B}$ and $R_{A}{ }^{B}$ each have a vanishing diagonal block, and so $S(a) \cong s o(5)$ [3].

We next consider the case $(\pi, V)=\left(\bigoplus_{r=1}^{t} \pi_{r}, \bigoplus_{r=1}^{t} V_{r}\right)$, for which the corresponding graph is a straight chain (all couplings being two-way). Thus, the linkages are either all Týpe I, with

$$
\begin{aligned}
k_{r+1} & =k_{r}+\frac{1}{2}, \\
l_{r+1} & =l_{r}-\frac{1}{2}, \quad 1 \leqslant r \leqslant t-1,
\end{aligned}
$$

or all Type II, with

$$
\begin{aligned}
k_{r+1} & =k_{r}+\frac{1}{2}, \\
l_{r+1} & =l_{r}+\frac{1}{2}, \quad 1 \leqslant r \leqslant t-1 .
\end{aligned}
$$

We know that $\left[T^{A B}(a)\right]$ corresponds to a point $a=\left(a_{1}, \ldots, a_{p}\right) \in \mathbf{C}^{p}$, where

$$
a_{r}=a_{r, r+1} a_{r+1, r}, \quad 1 \leqslant r \leqslant p=t-1 .
$$

By Theorem 3.3, $S(a) \subseteq s p(V)(s o(V))$, for any choice of $T^{A B}(a)$. It is possible to generalize the method of proof of Theorem 3.4 for "almost all" choices of $a=\left(a_{1}, \ldots, a_{p}\right)$. More precisely, the proof is valid for every $a \in D$, where $D$ is a subset of $\mathbf{C}^{p}$ which is dense in the Zariski topology on $\mathbf{C}^{p}[15]$.

THEOREM 3.5. Let $V$ be as above, such that the graph of $V$ does not meet the $k$ or $l$ axes. Then $S(a)=s p(V)(s o(V))$ for every $a \in D, D \subseteq \mathbf{C}^{p}$ being defined below:

Proof (I). Suppose the linkages are all Type I. For any $V$, it is clear that $\left(r\left|R_{A}{ }^{B}\right| s\right)$ and $\left(r\left|R_{A}{ }^{B}\right| s\right)$ can be non-zero only if $k_{s}=k_{r}$ and $l_{s}=l_{r}, l_{r} \pm 1$ or if $k_{s}=k_{r}, k_{r} \pm 1$ and $l_{s}=l_{r}$. Thus, for a straight chain, only the diagonal blocks $\left(r\left|R_{A}{ }^{B}\right| r\right),\left(r\left|R_{A}{ }^{B}\right| r\right)$ can be non-zero. By using (3.8) and (3.11), we obtain:

$$
\left(r\left|R_{A}^{B}\right| r\right)=g_{r}(a) K_{A}^{B}\left(k_{r}\right), \quad\left(r\left|R_{A}^{B}\right| r\right)=h_{r}(a) L_{A}^{B}\left(l_{r}\right),
$$

where, for $1 \leqslant r \leqslant t, g_{r}(a)$ and $h_{r}(a)$ are defined by

$$
\begin{aligned}
& g_{r}(a)=\frac{1}{2}\left\{-\left(2 l_{r-1}+1\right) a_{r-1}+2 l_{r} a_{r}\right\}, \\
& h_{r}(a)=\frac{1}{2}\left\{2 k_{r} a_{r-1}-\left(2 k_{r}+2\right) a_{r}\right\} .
\end{aligned}
$$

We now take

$$
D=\left\{a \in \mathbf{C}^{p} \mid g_{r}(a) \neq g_{s}(a), h_{r}(a) \neq h_{s}(a) \text { for all } r \neq s\right\} .
$$

Choose $a \in D$ : the coefficients $g_{r}(a)$ are then all distinct, and so are the $h_{r}(a)$. Also 
by hypothesis, $k_{1}>0$ and $l_{s}>0$, so that $K_{A}{ }^{B}\left(k_{r}\right), L_{A}{ }^{B}\left(l_{r}\right)$ are non-zero, for $1 \leqslant r \leqslant t$. Thus, the linear span $R$ of the matrices $R_{A}{ }^{B}$ is of the form

$$
R=\left\{\gamma(x)=\bigoplus_{r=1}^{t} \xi_{r} \pi_{r}(x) \mid x \in s l(2, \mathbf{C})\right\}
$$

where the $\xi_{r}$ are all distinct. If $x, y \in s l(2, \mathbf{C})$, we have

$$
[\gamma(x), \gamma(y)]=\bigoplus_{r=1}^{\ell} \xi_{r}^{2} \pi_{r}([x y]),
$$

and since $\operatorname{sl}(2, \mathrm{C})$ is semi-simple we therefore can generate the matrices

$$
\gamma^{(1)}(x)=\bigoplus_{r=1}^{t} \xi_{r}^{2} \pi_{r}(x), \quad x \in \operatorname{sl}(2, \mathbf{C}) .
$$

More generally, we can generate

$$
\gamma^{(n)}(x)=\bigoplus_{r=1}^{\ell} \xi_{r}^{n-1} \pi_{r}(x), \quad x \in \operatorname{sl}(2, \mathbf{C})
$$

Clearly, for $x \in \operatorname{si}(2, \mathrm{C})$, the matrices $\gamma(x), \gamma^{(1)}(x), \gamma^{(2)}(x)$, etc. go over all the linearly independent matrices of the form

$$
\rho(x)=\bigoplus_{r=1}^{t} \eta_{r} \pi_{r}(x), \quad \eta_{r} \in \mathbf{C} .
$$

Thus, in particular, we see that $S(a)$ contains the matrices

$$
\Delta_{i}(x)=0 \oplus \ldots \oplus \pi_{i}(x) \oplus 0 \oplus \ldots \oplus 0, \quad x \in \operatorname{sl}(2, \mathrm{C})
$$

( $\pi_{i}(x)$ occurring as the $i$ th factor). In fact, this result holds for all $x \in \operatorname{so}(4, \mathrm{C})$, as we can see by considering the linear span $R^{\prime}$ of the matrices $R_{A}{ }^{B}$.

Now put

$$
T^{1 \mathrm{i}}=\left[\begin{array}{cccccc}
0 & X_{12} & & & \\
\tilde{X}_{12} & 0 & X_{23} & & \\
& \tilde{X}_{23} & 0 & \ddots & \\
& & \ddots & \ddots & \ddots & \\
& & & \ddots & \ddots & X_{t-1, t} \\
& 0 & & \ddots & \ddots & \\
& & & & X_{t-1, t} & 0
\end{array}\right]
$$

Then, by examining the commutators $\left(\operatorname{ad} \Delta_{r}(x)\right)^{m}\left(\operatorname{ad} \Delta_{r+1}(y)\right)^{n} T^{1 \mathrm{i}}\left(x, y \in D_{2}\right.$, 
$\left.m, n \in \mathbf{Z}^{+}\right)$, for $1 \leqslant r \leqslant t-1$, we find that $S(a)$ contains the matrices

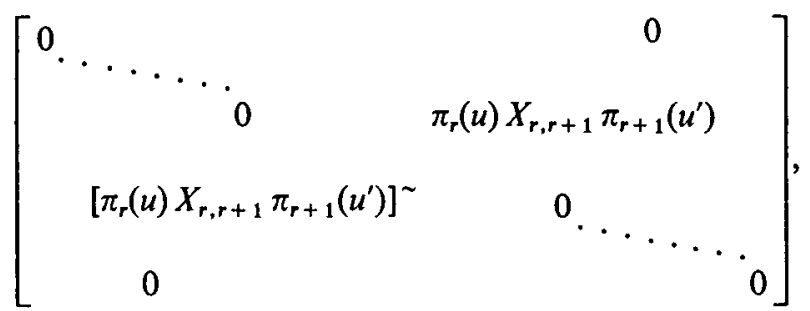

where $u, u^{\prime} \in U \backslash\{1\}$. Arguing as in the proof of Theorem 3.4, we see that $S(a)$ contains all matrices with an arbitrary $r, r+1$ block and all other blocks zero (except for the $r+1, r$ block), for $1 \leqslant r \leqslant t-1$. It is easy to show that such matrices are enough to generate all of $s p(V)(s o(V))$, and so the result follows.

(II) If the linkages are all Type II, and we assume that $k_{1}, l_{1}>0$, the argument is analogous to (I), with the appropriate choice of $D \subseteq \mathrm{C}^{p}$.

It is probable that Theorem 3.5 remains valid for all $a \in \mathbf{C}^{p}$. This may be proved directly for the case $t=3$, but the argument is long and unilluminating, and does not appear to generalize to $t>3$, so we omit it.

The situation is even worse for the most general $V$; usually, $R_{A}{ }^{B}$ and $R_{A}{ }^{B}$ will have non-vanishing off-diagonal matrix blocks, making $S(a)$ harder to calculate.

However, Theorems 3.4 and 3.5 cover a large class of wave equations. The Lie algebra approach we have used brings some order into the plethora of possible wave equations, because it tells us that certain wave equations with complicated graphs (and possibly repeated representations) are in fact members of the family of wave equations associated with some equation covered by Theorem 3.4 or 3.5. For example, if $V_{1}=\left(0, \frac{1}{2}\right), V_{2}=\left(\frac{1}{2}, 1\right), V_{3}=\left(1, \frac{1}{2}\right)$ and $V_{4}=\left(\frac{1}{2}, 0\right)$, and we take the closed chain $V_{1} \rightleftharpoons V_{2} \rightleftharpoons V_{3} \rightleftharpoons V_{4} \rightleftharpoons V_{1}$, then it was shown by Bhabha [3] that there is a unique choice of $T^{A B}$ (a) for which the off-diagonal blocks of $R_{A}{ }^{B}, R_{A}^{B}$ all vanish, and $S(a) \cong S o(5)$. For other choices of $T_{A 2}(a)$, of course, $S(a)$ cannot be isomorphic to $s o(5)$; amongst these other possibilities we have the Pauli-Fierz equation [8], and if we take $a_{12}=a_{23}=a_{34}=a_{41}=0$ we obtain the example $V_{1} \rightarrow V_{2} \rightarrow V_{3} \rightarrow V_{4} \rightarrow V_{1}$ mentioned earlier. In this case a tedious calculation shows that $S(a)=s l(16)$.

Given some wave equation for which $S(a)$ is known, we can in principle proceed to find the properties of the corresponding family of wave equations by obtaining the branching rules for $S(a) \rightarrow s o(4)$. However, we note that for $S(a)=D_{t}$ (and $\left.E_{7}, E_{8}\right)$, the embedding $s o(4) \subset S(a)$ is not uniquely specified, since we only know the branching rule for the natural representation. In fact, the embedding may a priori be one of two inequivalent types [12]; this ambiguity is very interesting, but we shall not consider it any further. In Section 5 , we discuss an example for which the embedding is uniquely specified. 
What sort of couplings may be expected in any given family of wave equations? If $\left(\pi_{2}, V(\lambda)\right)$ is an irreducible representation of $S(a)$ (with highest weight $\left.\lambda.\right)$ which is self-contragredient, then it is either symplectic or orthogonal, that is

$$
\pi_{\dot{\lambda}}(S(a)) \subseteq \operatorname{sp}(V(\lambda))(s o(V(\lambda))) .
$$

So it is clear that if there is a coupling, it must be two-way. However, not all the linked so(4)-sub-modules of $V(\lambda)$ need be coupled: only a sufficient number to ensure irreducibility. The representations of $s p(n)$ and $s o(2 n+1)$ are all selfcontragredient, but if $S(a)=s l(n)$ or $s o(2 n)$ this is not so, and one-way couplings may appear.

Finally, we consider the properties of the wave equation (1.1) in which $\kappa$ may be any matrix. It is clear that for relativistic invariance, we must have (as well as (1.3))

$$
\left[\kappa, I^{\mu v}\right]=0 .
$$

As in (3.15), $\kappa$ must be of the form

$$
\kappa=\bigoplus_{r=1}^{k}\left(\kappa_{r} \otimes I_{d_{r}}, \quad \kappa_{r} \in g l\left(n_{r}\right) .\right.
$$

It is natural to consider the Lie algebra $K(a)$ generated by $S(a)$ and $\kappa$. We then have the following general result.

THEOREM 3.6. Suppose $\kappa$ is not a multiple of the identity. If $\pi$ has no repeated sub-representations, and $S(a)=s p(V)(s o(V))$, then $K(a)=s l(V)$ if $T r \kappa=0$ and $K(a)=g l(V)$ if $\operatorname{Tr} \kappa \neq 0$.

Proof. The $r, s$ block of the commutator $\left[\kappa, T^{A \dot{B}}(a)\right]$ will be

while the $s, r$ block is

$$
\left(\kappa_{r}-\kappa_{s}\right)\left(r \mid T^{A B}(a \mid s),\right.
$$

$$
-\left(\kappa_{r}-\kappa_{s}\right)\left(s\left|T^{A \dot{B}}(a)\right| r\right) \text {. }
$$

Since $\kappa$ is not a multiple of the identity, there exist $r, s$ such that $\kappa_{r} \neq \kappa_{s}$, and clearly $\left[\kappa, T^{A B}(a)\right] \in s p(V)(s o(V))$ when $T^{A B}(a) \in s p(V)(s o(V))$. Now the Lie algebra $K^{\prime}(a)$ generated by $S(a)$ and $\left[\kappa, T^{A B}(a)\right]$ consists of trace zero matrices, acts irreducibly on $V$, and contains $S(a)$ properly. Since $s p(V)$ and $s o(V)$ are maximal among the semi-simple sub-algebras of $s l(V)$, it is clear that $K^{\prime}(a)=s l(V)$. Thus $K(a)=s l(V)$ if $\kappa$ is traceless: otherwise $K(a)=g l(V)$.

To take a well-known example, if $\pi=\left(\frac{1}{2}, 0\right) \oplus\left(0, \frac{1}{2}\right)$ (the Dirac equation), then $S=s p(4) \cong s o(5)$ and if

$$
\kappa=\left[\begin{array}{cc}
\kappa_{1} I_{2} & 0 \\
0 & \kappa_{2} I_{2}
\end{array}\right], \quad \kappa_{1}, \kappa_{2} \in \mathbf{C},
$$

then $K=s l(4) \cong s o(6)$ if $\kappa_{2}=-\kappa_{1}$, otherwise $K=g l(4)$. 


\section{The discrete transformations. Reality conditions}

Suppose we have a wave equation of the form (1.1), invariant under the proper Lorentz group; thus, as in Section 3, we have a representation

$$
(\pi, V)=\left(\bigoplus_{i=1}^{t} \pi_{i}, \bigoplus_{i=1}^{t} V_{i}\right)
$$

of $s o(4, \mathrm{C})$, and a vector operator $T^{A B}(a)$. We now consider the equation of the existence of operators $P, C, T$, corresponding to space reflection, charge conjugation and time reversal, respectively. The further assumption that (1.1) is invariant under these transformations gives conditions on the $a_{r s}$. The Lie algebra structure is not important here; in fact we must treat separately each member of the class of wave equations corresponding to some Lie algebra $L \supset \operatorname{so}(4, \mathrm{C})$, appending the operators $P, C, T$ "by hand" in each case.

In order to simplify the formulae, we shall assume throughout this section that $\pi$ has no repeated representations. It is not hard to derive the analogous formulae for the general case.

\section{(1) Space reflection}

As is well known, if we define

then we must have

$$
\begin{aligned}
\psi^{P}\left(x^{\prime}\right) & =P \psi(x), \\
x^{0 \prime} & =x^{0}, \quad x^{\prime}=-x,
\end{aligned}
$$

$$
P K_{j}=L_{j} P
$$

It follows that the matrix blocks of $P$ are of the form

$$
(r|P| s)_{m_{r} n_{r} ; m_{s} n_{s}}=\omega(r) \delta_{r \bar{s}} \delta_{m_{r} n_{s}} \delta_{n_{r} m_{s}} .
$$

Here, $\delta_{r s}$ means $\delta_{k_{r} k_{s}} \delta_{l_{r} l_{s}}$, and $\bar{r}$ will always refer to the conjugate $\left(l_{r}, k_{r}\right)$ of the representation $\left(k_{r}, I_{r}\right) ; \omega(r)$ is given in the table below

\begin{tabular}{ccc}
\hline & $P^{2}=I$ & $P^{2}=-I$ \\
\hline$k_{r} \neq l_{r}$ & 1 & $i$ \\
$k_{r}=l_{r}$ & \pm 1 & $\pm i$ \\
\hline
\end{tabular}

Thus, $P$ exists if with each irreducible sub-representation $\pi_{r}$ of $\pi$, the conjugate $\bar{\pi}_{r}$ also appears in $\pi$.

The wave equation (1.1) is invariant under spatial reflection if

$$
P \alpha^{0}=\alpha^{0} P, \quad P \alpha^{k}=-\alpha^{k} P .
$$

It is sufficient to consider the condition on $\alpha^{0}$; the conditions on $\alpha^{k}$ will then follow 
from (4.2). Since $\alpha^{0}=\frac{1}{2}\left(T^{1 \mathrm{i}}+T^{2 \dot{2}}\right)$, if we substitute (3.7) and (4.3) into the equation

we obtain

$$
P\left(T^{1 \mathrm{i}}+T^{2 \hat{z}}\right)=\left(T^{1 \mathrm{i}}+T^{2 \dot{z}}\right) P,
$$

$$
\omega(r) a_{\bar{r} s}=-a_{r \bar{s}} \omega(\bar{s}) .
$$

Replacing $s$ by $\bar{s}$, we may write

$$
\omega(s)=-\frac{a_{\bar{r} \bar{s}}}{a_{r s}} \omega(r)
$$

Clearly, we have

$$
\begin{array}{ll}
a_{\bar{r} \bar{s}}=-a_{r s} & \text { if } k_{r} \neq l_{r}, k_{s} \neq l_{s}, \\
a_{\bar{r} s}=\mp a_{r s} & \text { if } k_{r} \neq l_{r}, k_{s}=l_{s},
\end{array}
$$

and if $k_{r}=l_{r}, k_{s}=l_{s}$, then there is no condition on $a_{r s}$, but $P$ must act in the opposite way on $V_{r}, V_{s}$.

If $\gamma$ is a closed loop of the form (3.17), then by eliminating $\omega\left(r_{1}\right)$ from the equations (4.6), we obtain the further condition:

$$
\frac{a_{\tilde{r}_{1} \tilde{r}_{2}} a_{\vec{r}_{2} \bar{r}_{3}} \ldots a_{\bar{r}_{n-1} \bar{r}_{n}}}{a_{r_{1} r_{2}} a_{r_{2} r_{3}} \ldots a_{r_{n-1} r_{n}}}=(-1)^{n} \frac{a_{\tilde{r}_{1} \tilde{r}_{n}}}{a_{r_{1} r_{n}}}=\frac{a_{\tilde{r}_{1} \tilde{r}_{n}}}{a_{r_{1} r_{n}}}
$$

(since $n$ must be even).

\section{(2) Charge conjugation}

We define [6b]

$$
\psi^{c}(x)=\mathscr{C} \psi(x)=C \bar{\psi}(x),
$$

where $\mathscr{C}$ is antilinear and $C$ linear, and require that $\psi^{C}$ transforms like $\psi$ under a Lorentz transformation $\Lambda$ :

$$
\psi^{c^{\prime}}\left(x^{\prime}\right)=\pi(\Lambda) \psi^{c}(x), \quad x^{\prime}=\Lambda x .
$$

This means that

$$
C \overline{\pi(\Lambda)}=\pi(\Lambda) C
$$

and so

$$
C K_{3}=-L_{3} C, \quad C K_{ \pm}=-L_{\mp} C .
$$

From (4.9), it is clear that the matrix blocks of $C$ are of the form:

$$
(r|C| s)_{m_{r} n_{r}: m_{s} n_{s}}=\zeta(r)(-1)^{k_{r}+m_{r}+l_{r}+n_{r}} \delta_{r s} \delta_{m_{r},-n_{s}} \delta_{n_{r},-m_{s}}, \quad \zeta(r) \in \mathrm{C} .
$$

The condition

$$
\psi^{c c}=\psi \quad(\text { that is } C \bar{C}=I)
$$

implies that

$$
\left.\zeta(r) \overline{\zeta(r)}=(-1)^{2\left(k_{r}+I_{r}\right)}\right)
$$


If we also require that $\psi^{c}$ transforms like $\psi$ under space reflection, then

$$
C \bar{P}=P C
$$

which means that

$$
\zeta(r) \overline{\omega(\bar{r})}=\omega(r) \zeta(\bar{r}) .
$$

Thus we see that (4.12) and (4.14) are compatible provided that we take $P^{2}=I$ for particles with integer spin, and $P^{2}=-I$ for particles with half-integer spin.

In the presence of an external electromagnetic field, with the assumption of minimal coupling, the wave equation (1.1) becomes

$$
\alpha^{\mu}\left(\frac{\partial}{\partial x^{\mu}}-i e A_{\mu}\right) \psi(x)+i \kappa \psi(x)=0,
$$

where $e$ is the charge. If we demand that $\psi^{c}$ satisfies

$$
\alpha^{\mu}\left(\frac{\partial}{\partial x^{\mu}}+i e A_{\mu}\right) \psi^{c}(x)+i \kappa \psi^{c}(x)=0
$$

then we obtain

$$
\alpha^{\mu} C+C \overline{\alpha^{\mu}}=0 .
$$

The condition for $\alpha^{0}$, on using (3.7) and (4.10), becomes

$$
\begin{aligned}
& (-1)^{k_{s}+l_{s}-m_{s}-n_{s}} \zeta(\bar{s}) a_{r \dot{s}} \sum_{\rho=\frac{1}{1},-\frac{1}{2}}(-1)^{\frac{1}{2}-\rho}\left(\frac{1}{2} l_{s} k_{r} m_{r} \mid-\rho-n_{s}\right)\left(\frac{1}{2} k_{s} l_{r} n_{r} \mid \rho-m_{s}\right) \\
& =-(-1)^{k_{r}+l_{r}+m_{r}+n_{r} \zeta(r) \bar{a}_{\tilde{r} s}} \sum_{\rho=\frac{1}{2},-\frac{1}{t}}(-1)^{\frac{1}{2}-\rho}\left(\frac{1}{2} k_{s} l_{r}-n_{r} \mid-\rho m_{s}\right)\left(\frac{1}{2} l_{s} k_{r}-m_{r} \mid \rho n_{s}\right) .
\end{aligned}
$$

If we use the identity

$$
\left(j_{1} j_{2} j m \mid m_{1} m_{2}\right)=(-1)^{j_{1}+j_{2}-j}\left(j_{1} j_{2} j-m \mid-m_{1}-m_{2}\right),
$$

and note that $k_{r}+l_{r}$ and $k_{s}+l_{s}$ are simultaneously integral or half-integral, we obtain (replacing $s$ by $\bar{s}$ ):

$$
\zeta(s)=\frac{\bar{a}_{\overline{r s}}}{a_{r s}} \zeta(r) .
$$

Again, if $\gamma$ is the closed loop (3.17), we must also have:

$$
\frac{\bar{a}_{\bar{r}_{1} \bar{r}_{2}} \bar{a}_{\bar{r}_{2} \bar{r}_{3} \ldots \bar{a}_{\bar{r}_{n-1} \bar{r}_{n}}}}{a_{r_{1} r_{2}} a_{r_{2} r_{3}} \ldots a_{r_{n-1} r_{n}}}=\frac{\bar{a}_{\bar{r}_{1} \bar{r}_{n}}}{a_{r_{1} r_{n}}} .
$$

\section{(3) Time reversal}

For physical reasons, we use the Wigner definition:

$$
\psi^{T}\left(x^{\prime}\right)=\mathscr{T} \psi(x)=T \psi(x), \quad x^{0 \prime}=-x^{0}, \quad x^{\prime}=x,
$$

where $\mathscr{T}$ is antilinear and $T$ linear. To obtain the multiplication relations for $T$ 
with the generators $I_{\mu \nu}$ of $\pi$, we observe that, since the orbital part of the angular momentum tensor is

$$
L_{\mu \nu}=i\left(x_{\mu} \frac{\partial}{\partial x^{\nu}}-x_{v} \frac{\partial}{\partial x^{\mu}}\right),
$$

then under time reversal we should have

$$
\begin{aligned}
& L_{k l} \rightarrow-L_{k l}, \\
& L_{0 k} \rightarrow L_{0 k} .
\end{aligned}
$$

The spin part is $J_{\mu \nu}=i I_{\mu v}$ and so, by analogy, we take

$$
\begin{aligned}
& \mathscr{T} J_{k l} \mathscr{T}^{-1}=-J_{k l}, \\
& \mathscr{T} J_{0 k} \mathscr{T}^{-1}=J_{0 k} .
\end{aligned}
$$

These relations give us

$$
\begin{array}{ll}
T K_{3}=-K_{3} T, & T K_{ \pm}=-K_{\mp} T, \\
T L_{3}=-L_{3} T, & T L_{ \pm}=-L_{\mp} T .
\end{array}
$$

The matrix blocks of $T$ thus are of the form:

$$
(r|T| s)_{m_{r} n_{r} ; m_{s} n_{s}}=\tau(r)(-1)^{k_{r}+I_{r}+m_{r}+n_{r}} \delta_{r s} \delta_{m_{r},-m_{s}} \delta_{n_{r},-n_{s}}, \quad \tau(r) \in \mathbf{C} .
$$

It is well known that

and this gives

$$
\mathscr{T}^{2}= \pm I, \text { that is } T \bar{T}= \pm I
$$

$$
|\tau(r)|^{2}(-1)^{2\left(k_{r}+t_{r}\right)}= \pm 1
$$

Thus, $\tau(r)$ is a phase factor, and we must take $\mathscr{T}^{2}=+I$ for integer spin, and $\mathscr{T}^{2}=-I$ for half-integer spin.

We turn now to the question of when (1.1) is invariant under time reversal. It is easy to see that this is so when

$$
T \overline{\alpha^{0}}-\alpha^{0} T=0, \quad T \overline{\alpha^{k}}+\alpha^{k} T=0 .
$$

Imitating the argument used for charge conjugation, we can use the condition on $\alpha^{0}$ to obtain

$$
\tau(s)=\frac{\bar{a}_{r s}}{a_{r s}} \tau(r) .
$$

Also, the further condition

$$
\frac{\bar{a}_{r_{1} r_{2}} \bar{a}_{r_{2} r_{3} \ldots \bar{a}_{r_{n-1} r_{n}}}}{a_{r_{1} r_{2}} a_{r_{2} r_{3}} \ldots a_{r_{n-1} r_{n}}}=\frac{\bar{a}_{r_{1} r_{n}}}{a_{r_{1} r_{1}}}
$$

must hold for the closed loop (3.17).

Finally, we discuss the conditions under which (1.1) is obtainable from an invariant Lagrangian [8]. We require the existence of a non-degenerate Hermitian 
form $h(v, w)$ on $V$, invariant under proper Lorentz transformations $\Lambda$ :

$$
\begin{aligned}
h\left(v, \alpha w_{1}+\beta w_{2}\right) & =\alpha h\left(v, w_{1}\right)+\beta h\left(v, w_{2}\right), \\
h\left(\alpha v_{1}+\beta v_{2}, w\right) & =\bar{\alpha} h\left(v_{1}, w\right)+\bar{\beta} h\left(v_{2}, w\right), \\
h(v, w) & =\overline{h(w, v)}, \\
h(\pi(\Lambda) v, \pi(\Lambda) w) & =h(v, w) .
\end{aligned}
$$

The last equation says that

$$
h\left(I^{\mu v} v, w\right)+h\left(v, I^{\mu v} w\right)=0
$$

or, if $H$ is the matrix of $h$ :

$$
H I^{\mu \nu}=-\left(I^{\mu v}\right)^{\dagger} H
$$

(where $A^{\dagger}$ means $\bar{A}^{\mathrm{T}}$ ). Thus

$$
\begin{aligned}
& H K_{3}=L_{3}^{\dagger} H=L_{3} H, \\
& H K_{ \pm}=L_{\mp}^{\dagger} H=L_{ \pm} H .
\end{aligned}
$$

Therefore $H$ has matrix blocks

$$
(r|H| s)_{m_{r} n_{r} ; m_{s} n_{s}}=h(r) \delta_{r \bar{s}} \delta_{m_{r} n_{s}} \delta_{n_{r} m_{s}}, \quad h(r)=\overline{h(\check{r})} .
$$

Such a form exists, then, if for each sub-representation $\pi_{r}$ of $\pi$, the conjugate $\pi_{r}$ also appears in $V$.

If $h$ is also to be invariant under space reflection, we have

$$
H=P^{\dagger} H P, \text { that is } h(r) \in \mathbf{R} .
$$

The wave equation (1.1) is derivable from an invariant Lagrangian if, in addition to the above [8],

or

$$
h\left(\alpha^{0} v, w\right)=h\left(v, \alpha^{0} w\right)
$$

$$
H \alpha^{o}=\left(\alpha^{0}\right)^{\dagger} H
$$

By substituting (3.7) and (4.31) into (4.32), we obtain

$$
h(\bar{s})=\varepsilon_{r s} \frac{\bar{a}_{\bar{r} \bar{s}}}{a_{s r}} h(\bar{r}),
$$

where $\varepsilon_{r s}=(-1)^{\left(k_{s}+l_{s}\right)-(k r+t r)}=+1$ or -1 according as $V_{r} \rightleftharpoons V_{s}$ is Type I or Type II. Furthermore, we see that for the closed loop (3.17):

$$
\frac{\bar{a}_{\bar{r}_{1} \bar{r}_{2}} \bar{a}_{\bar{r}_{2} \bar{r}_{3} \ldots \bar{a}_{\bar{r}_{n-1} \bar{r}_{n}}}}{a_{r_{2} r_{1}} a_{r_{3} r_{2}} \ldots a_{r_{n} r_{n-1}}}=\frac{\bar{a}_{\bar{r}_{1} \bar{r}_{n}}}{a_{r_{n} r_{1}}}
$$

(the factors $\varepsilon_{r s}$ cancel).

It is interesting to observe here that, if we assume the wave equation (1.1) 
to be invariant under charge conjugation and also obtainable from an invariant Lagrangian, then by combining (4.19) and (4.34) we have, for every closed loop $\gamma$,

$$
a^{+}(\gamma)=a^{-}(\gamma)
$$

But this is exactly the condition that $T^{A B}(a)$ be skew relative to some form $B$ (by Theorem 3.3). There is therefore some physical justification for taking $S(a)$ to be a sub-algebra of $s p(V)(s o(V))$.

Finally, if we demand locality of the theory, then the equation (1.1) must be invariant under the combined operation $\theta=P \mathscr{C} \mathscr{T}=P C \bar{T}$. Since $\theta$ has matrix blocks

where

$$
(r|\theta| s)_{m_{r} n_{r} ; m_{s} n_{s}}=\kappa(r) \delta_{r s} \delta_{m_{r} m_{s}} \delta_{n_{r} n_{s}}
$$

$$
\kappa(r)=(-1)^{\left(2 k_{r}+l_{r}\right)} \omega(r) \zeta(\bar{r}) \tau(r),
$$

we have invariance under $\theta$ if

$$
\theta \alpha^{\mu}+\alpha^{\mu} \theta=0, \text { that is } \kappa(r)=-\kappa(s) .
$$

The phases of $P, \mathscr{C}, \mathscr{T}$ are easily chosen to satisfy (4.36).

\section{The Kursunoğlu equation}

Throughout this section, we shall take $(\pi, V)=\left(\pi_{1} \oplus \pi_{2}, V_{1} \oplus V_{2}\right)$, with $\pi_{1}=\left(1, \frac{1}{2}\right)$, $\pi_{2}=\left(\frac{1}{2}, 1\right)$. The corresponding wave equation will be referred to as the Kursunoglu equation, although it is not the most general form of his equation [13]. By Theorem 3.4 , we know that $S(a)=s p(V)=s p(12)$, no matter how we choose the vector operator $T^{A B}\left(a_{12}, a_{21}\right)$. The Kursunoglu equation gives rise to a whole family of wave equations: one for each irreducible representation of $s p(12)$.

First, we consider the Lorentz content of some of these equations, We must give the branching rules for

or

$$
s p(12) \supset s p(6) \oplus s p(6) \supset s o(4)
$$

$$
C_{6} \supset C_{3} \oplus C_{3} \supset D_{2},
$$

where the branching rule for the natural representation is

$$
(100000) \rightarrow(100 ; 000) \oplus(000 ; 100) \rightarrow\left(1, \frac{1}{2}\right) \oplus\left(\frac{1}{2}, 1\right) .
$$

From Section 2, we know that (5.2) specifies the embedding $D_{2} \subset C_{6}$ up to equivalence.

The branching rules for $C_{6} \supset C_{3} \oplus C_{3}$ are readily obtained from the formula given in the proof of Proposition 3.2. In Table 1 we give a list of these branching rules for the lowest dimensional irreducible representations of $C_{6}$, where we have made use of the tables for the division of $S$-functions given in [19]. Note that if one of the arguments of the term $\langle\alpha ; \beta\rangle$ involves a Young diagram with more than 
three rows, we must use the modification rules [16] to express $\langle\alpha ; \beta\rangle$ in terms of simpler quantities involving Young diagrams with three rows or less.

To obtain the branching rules for $C_{3} \oplus C_{3} \rightarrow D_{2}$, we need to make full use of Dynkin's method. Consider the embedding

$$
\begin{gathered}
C_{3} \supset D_{2}, \\
(100) \rightarrow\left(1, \frac{1}{2}\right) .
\end{gathered}
$$

The weights of the representation $\left(1, \frac{1}{2}\right)$ are

$$
\begin{aligned}
& \mu_{1}^{\prime}=2 \lambda_{1}^{\prime}+\lambda_{2}^{\prime}, \\
& \mu_{2}^{\prime}=2 \lambda_{1}^{\prime}-\lambda_{2}^{\prime}, \\
& \mu_{3}^{\prime}=\lambda_{2}^{\prime}, \\
& \mu_{4}^{\prime}=-\lambda_{2}^{\prime}, \\
& \mu_{5}^{\prime}=-2 \lambda_{1}^{\prime}+\lambda_{2}^{\prime}, \\
& \mu_{6}^{\prime}=-2 \lambda_{1}^{\prime}-\lambda_{2}^{\prime} .
\end{aligned}
$$

TABLE 1

Some branching rules for $C_{6} \rightarrow C_{3} \oplus C_{3}$ (see [19])

\begin{tabular}{lrl}
\hline$\langle\lambda\rangle$ & $\operatorname{dim} V(\lambda)$ & \multicolumn{1}{c}{ Branching rule } \\
\hline$\langle 0\rangle$ & 1 & $\langle 0 ; 0\rangle$ \\
$\langle 1\rangle$ & 12 & $\langle 1 ; 0\rangle \oplus\langle 0 ; 1\rangle$ \\
$\left\langle 1^{2}\right\rangle$ & 65 & $\left\langle 1^{2} ; 0\right\rangle \oplus\left\langle 0 ; 1^{2}\right\rangle \oplus\langle 1 ; 1\rangle \oplus\langle 0 ; 0\rangle$ \\
$\langle 2\rangle$ & 78 & $\langle 2 ; 0\rangle \oplus\langle 0 ; 2\rangle \oplus\langle 1 ; 1\rangle$ \\
$\left\langle 1^{3}\right\rangle$ & 208 & $\left\langle 1^{3} ; 0\right\rangle \oplus\left\langle 0 ; 1^{3}\right\rangle \oplus\left\langle 1^{2} ; 1\right\rangle \oplus\left\langle 1 ; 1^{2}\right\rangle \oplus\langle 1 ; 0\rangle \oplus\langle 0 ; 1\rangle$ \\
$\langle 3\rangle$ & 364 & $\langle 3 ; 0\rangle \oplus\langle 0 ; 3\rangle \oplus\langle 2 ; 1\rangle \oplus\langle 1 ; 2\rangle$ \\
$\left\langle 1^{4}\right\rangle$ & 429 & $\left\langle 1^{3} ; 1\right\rangle \oplus\left\langle 1 ; 1^{3}\right\rangle \oplus\left\langle 1^{2} ; 1^{2}\right\rangle \oplus\left\langle 1^{2} ; 0\right\rangle \oplus\left\langle 0 ; 1^{2}\right\rangle \oplus\langle 1 ; 1\rangle \oplus\langle 0 ; 0\rangle$ \\
$\left\langle 1^{6}\right\rangle$ & 429 & $\left\langle 1^{3} ; 1^{3}\right\rangle \oplus\left\langle 1^{2} ; 1^{2}\right\rangle \oplus\langle 1 ; 1\rangle \oplus\langle 0 ; 0\rangle$ \\
$\langle 21\rangle$ & 560 & $\langle 21 ; 0\rangle \oplus\langle 0 ; 21\rangle \oplus\langle 2 ; 1\rangle \oplus\langle 1 ; 2\rangle \oplus\left\langle 1^{2} ; 1\right\rangle \oplus\left\langle 1 ; 1^{2}\right\rangle \oplus\langle 1 ; 0\rangle \oplus\langle 0 ; 1\rangle$ \\
$\left\langle 1^{3}\right\rangle$ & 572 & $\left\langle 1^{3} ; 1^{2}\right\rangle \oplus\left\langle 1^{2} ; 1^{3}\right\rangle \oplus\left\langle 1^{2} ; 1\right\rangle \oplus\left\langle 1 ; 1^{2}\right\rangle \oplus\langle 1 ; 0\rangle \oplus\langle 0 ; 1\rangle$ \\
$\left\langle 2^{2}\right\rangle$ & 1650 & $\left\langle 2^{2} ; 0\right\rangle \oplus\left\langle 0 ; 2^{2}\right\rangle \oplus\langle 21 ; 1\rangle \oplus\langle 1 ; 21\rangle \oplus\langle 2 ; 2\rangle \oplus\left\langle 1^{2} ; 1^{2}\right\rangle \oplus\left\langle 1^{2} ; 0\right\rangle \oplus\left\langle 0 ; 1^{2}\right\rangle$ \\
& & \\
\end{tabular}

These are ordered so that $\mu_{1}^{\prime} \geqslant \ldots \geqslant \mu_{6}^{\prime}$ relative to the ordering induced by the simple roots $\alpha_{1}^{\prime}=2 \lambda_{1}^{\prime}$ and $\alpha_{2}^{\prime}=2 \lambda_{2}^{\prime}$ of $D_{2}=A_{1} \oplus A_{1}$. The map $f^{*}$ is given by [12]:

$$
\begin{aligned}
& f^{*}\left(\alpha_{1}\right)=\mu_{1}^{\prime}-\mu_{2}^{\prime}=2 \lambda_{2}^{\prime}, \\
& f^{*}\left(\alpha_{2}\right)=\mu_{2}^{\prime}-\mu_{3}^{\prime}=2 \lambda_{1}^{\prime}-2 \lambda_{2}^{\prime}, \\
& f^{*}\left(\alpha_{3}\right)=\mu_{3}^{\prime}-\mu_{4}^{\prime}=2 \lambda_{2}^{\prime},
\end{aligned}
$$

or, equivalently, by [14]:

$$
\begin{aligned}
& f^{*}\left(\lambda_{1}\right)=\mu_{1}^{\prime}=2 \lambda_{1}^{\prime}+\lambda_{2}^{\prime}, \\
& f^{*}\left(\lambda_{2}\right)=\mu_{1}^{\prime}+\mu_{2}^{\prime}=4 \lambda_{1}^{\prime} \\
& f^{*}\left(\lambda_{3}\right)=\mu_{1}^{\prime}+\mu_{2}^{\prime}+\mu_{3}^{\prime}=4 \lambda_{1}^{\prime}+\lambda_{2}^{\prime}
\end{aligned}
$$


If now $V(\lambda)$ is an irreducible $C_{3}$-module, with $\lambda=\sum_{i=1}^{3} m_{i} \lambda_{i}$, and we know the full set $\Pi(\lambda)$ of weights of $V(\lambda)$, then by applying the map $f^{*}$ according to (5.5) or (5.6) we can immediately find the branching rules for (5.3). In the Appendix, we shall indicate in general how these weights and their multiplicities may be calculated, but for now we are content to use the weight diagrams for the lowest dimensional $C_{3}$-modules already given by Konuma, Shima and Wada [21]. The branching rules are given in Table 2, where both the notations $\left(m_{1}, m_{2}, m_{3}\right)$ and $\left\langle r_{1}, r_{2}, r_{3}\right\rangle$ for $V(\lambda)$ are used.

It is clear that the branching rules for the embedding

$$
\begin{gathered}
C_{3} \supset D_{2}, \\
(100) \rightarrow\left(\frac{1}{2}, 1\right),
\end{gathered}
$$

may be obtained from those for $(5.3)$ by writing $(l, k)$ instead of the $(k, l)$ occurring in Table 2.

We now observe that, since the irreducible $C_{3} \oplus C_{3}$-modules are of the form $V(\lambda) \otimes V(\bar{\lambda})$, with $V(\lambda), V(\bar{\lambda})$ irreducible $C_{3}$-modules, every weight $\mu$ of $V(\lambda) \otimes V(\bar{\lambda})$ is of the form $v+\bar{v}$, where $v(\bar{v})$ is a weight of $V(\lambda)(V(\bar{\lambda}))$. Thus $f^{*}(\mu)=f^{*}(v)+f^{*}(\bar{v})$, where $f^{*}(v)$ is specified from (5.3), and $f^{*}(\bar{v})$ from (5.7), and so, to obtain the branching rule for $C_{3} \oplus C_{3} \rightarrow D_{2}$, we take the tensor product of $V(\lambda)$ regarded as a $D_{2^{-}}$module from (5.3) with $V(\bar{\lambda})$ regarded as a $D_{2}$-module from (5.7) In Table 3, we give some of these branching rules, listing only those $\langle\alpha ; \beta\rangle$ with $\alpha, \beta \neq 0$; the cases where one or both of $\alpha, \beta$ are zero are covered by Table 2 . Also, we only list one of the pair $\langle\alpha ; \beta\rangle,\langle\beta ; \alpha\rangle$, since the branching rules are related by interchanging $(k, l)$ with $(l, k)$.

By combining these results, it is easy to find the branching rules (5.1) for all the irreducible representations of $s p(12)$ listed in Table 1. In Fig. 1, we give diagrams

TABLE 2

\begin{tabular}{|c|c|c|c|}
\hline \multicolumn{2}{|c|}{$V(\lambda)$} & \multirow[b]{2}{*}{$\operatorname{dim} V(\lambda)$} & \multirow[b]{2}{*}{ Branching rule } \\
\hline$\left(m_{1}, m_{2}, m_{2}\right)$ & $\left.r_{1}, r_{2}, r_{2}\right\rangle$ & & \\
\hline$(000)$ & $\langle 0\rangle$ & 1 & $(0,0)$ \\
\hline$(100)$ & $\langle 1\rangle$ & 6 & $\left(1, \frac{1}{2}\right)$ \\
\hline$(010)$ & $\left\langle 1^{2}\right\rangle$ & 14 & $(2,0) \oplus(1,1)$ \\
\hline$(001)$ & $\left\langle 1^{3}\right\rangle$ & 14 & $\left(2, \frac{1}{2}\right) \oplus\left(0, \frac{3}{2}\right)$ \\
\hline$(200)$ & $\langle 2\rangle$ & 21 & $(2,1) \oplus(1,0) \oplus(0,1)$ \\
\hline$(300)$ & $\langle 3\rangle$ & 56 & $\left(3, \frac{3}{2}\right) \oplus\left(2, \frac{1}{2}\right) \oplus\left(1, \frac{3}{2}\right) \oplus\left(1, \frac{1}{2}\right)$ \\
\hline$(110)$ & $\langle 21\rangle$ & 64 & $\left(3, \frac{1}{2}\right) \oplus\left(2, \frac{3}{2}\right) \oplus\left(2, \frac{1}{2}\right) \oplus\left(1, \frac{3}{2}\right) \oplus\left(1, \frac{1}{2}\right) \oplus\left(0, \frac{1}{2}\right)$ \\
\hline$(101)$ & $\left\langle 21^{2}\right\rangle$ & 70 & $(3,1) \oplus(3,0) \oplus(2,1) \oplus(1,2) \oplus(1,1) \oplus(1,0)$ \\
\hline$(002)$ & $\left\langle 2^{3}\right\rangle$ & 84 & $(4,1) \oplus(2,2) \oplus(3,0) \oplus(0,3) \oplus(2,1) \oplus(0,1)$ \\
\hline$(020)$ & $\left\langle 2^{2}\right\rangle$ & 90 & $(4,0) \oplus(3,1) \oplus(2,2) \oplus(2,1) \oplus(2,0) \oplus(1,1) \oplus(0,2) \oplus(0,0)$ \\
\hline
\end{tabular}

Some branching rules for $C_{3} \rightarrow D_{2}$, where $(100) \rightarrow\left(1, \frac{1}{2}\right)$ (see [16]) 

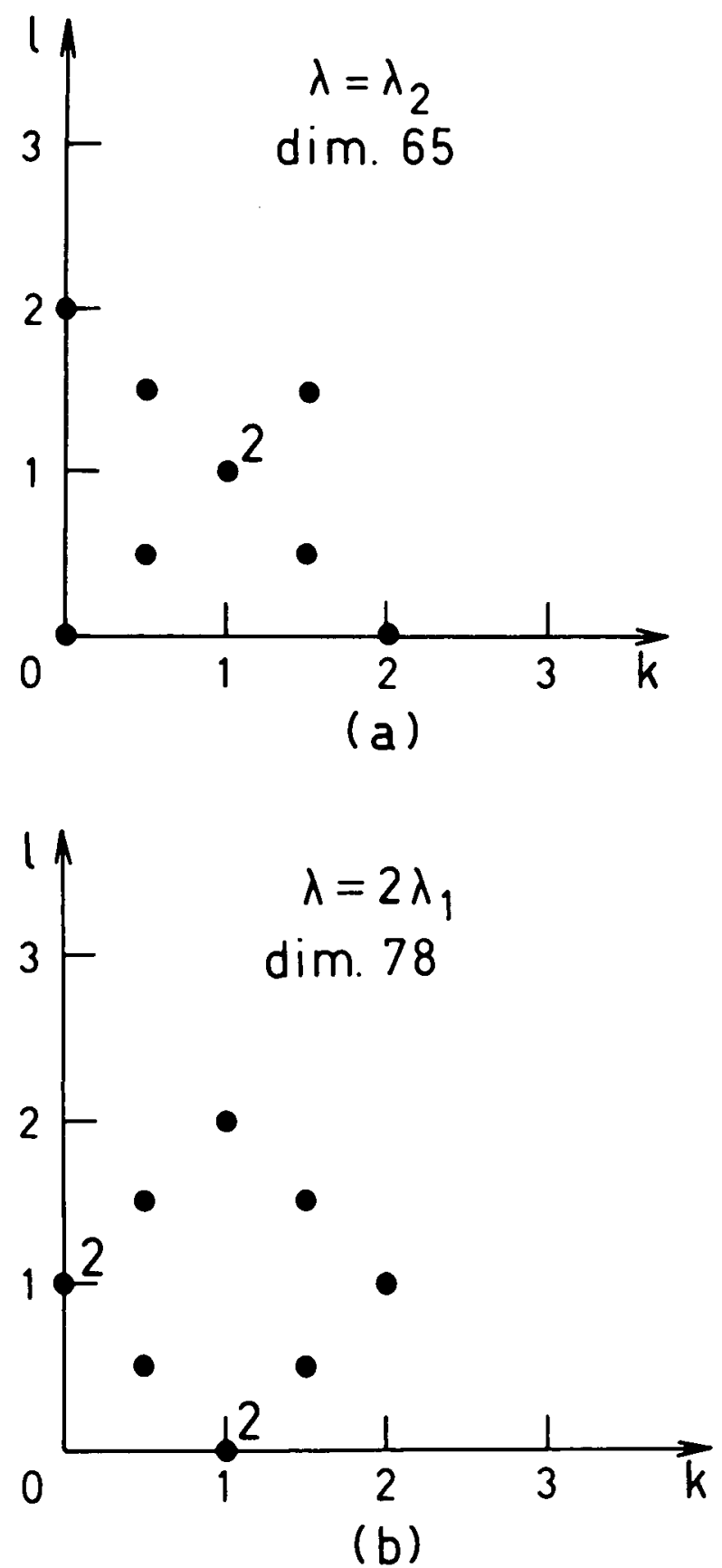

Fig. 1. Some members of the Kursunoğlu family of wave equations. Each graph represents the branching rule for some irreducible representation $V(\lambda)$ of $\operatorname{sp}(12)$, restricted to so(4). If an irreducible 

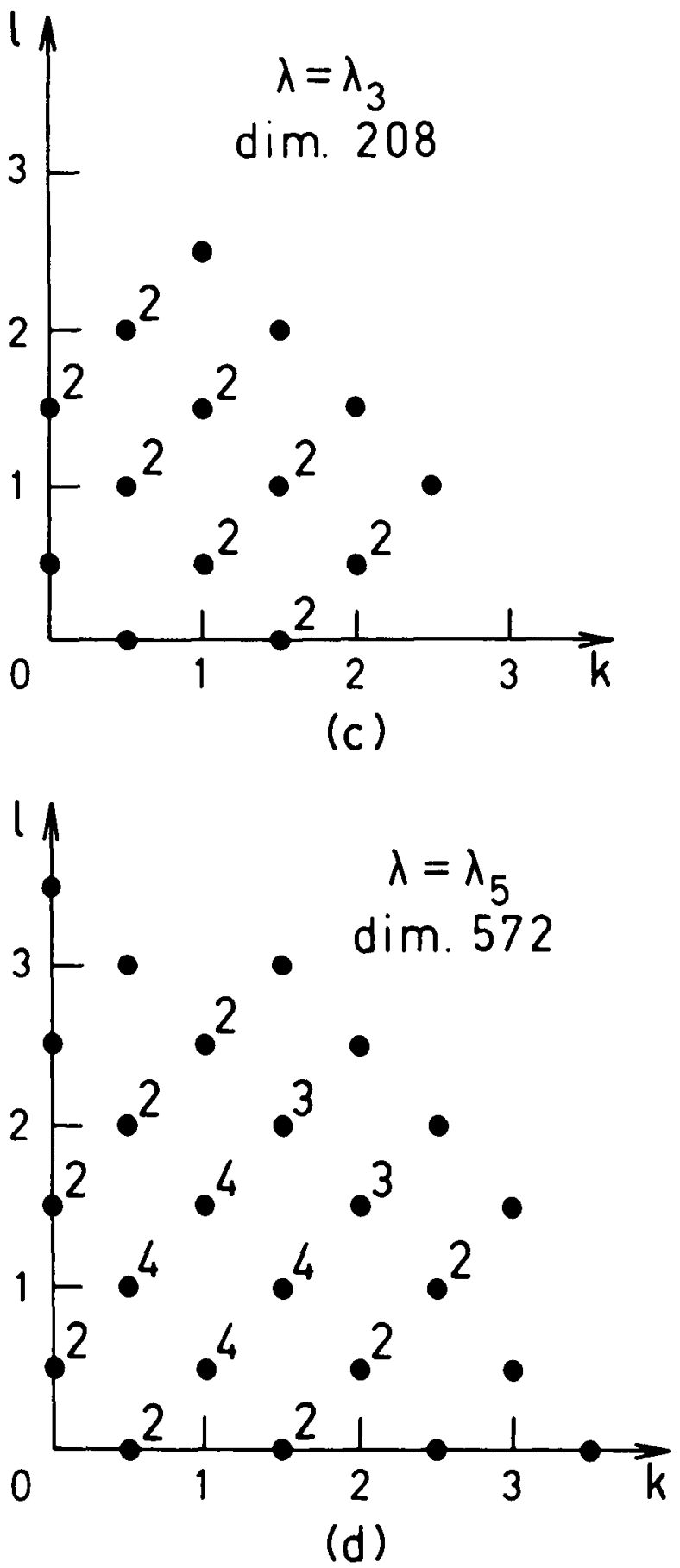

representation $(k, l)$ of $s o(4)$ occurs more than once, its multiplicity is shown on the diagram next to the point $(k, l)$. 
illustrating these rules for the representations with highest weights $\lambda_{2}=\lambda_{2}, \lambda=2 \lambda_{1}$ (the adjoint representation), $\lambda=\lambda_{3}$ and $\lambda=\lambda_{5}$.

TABLE 3

Some branching rules for $C_{3} \oplus C_{3} \rightarrow D_{2}$

\begin{tabular}{|c|c|c|}
\hline$V(\lambda) \otimes V(\lambda)$ & Dimension & Branching rule \\
\hline$\langle 1 ; 1\rangle$ & 36 & $\left(1, \frac{1}{2}\right) \otimes\left(\frac{1}{2}, 1\right) \cong\left(\frac{3}{2}, \frac{3}{2}\right) \oplus\left(\frac{3}{2}, \frac{1}{2}\right) \oplus\left(\frac{1}{2}, \frac{3}{2}\right) \oplus\left(\frac{1}{2}, \frac{1}{2}\right)$ \\
\hline$\left\langle 1^{2} ; 1\right\rangle$ & 84 & $\begin{array}{l}{[(2,0) \oplus(1,1)] \Theta\left(\frac{1}{2}, 1\right) \cong\left(\frac{3}{2}, 1\right) \oplus 2\left(\frac{3}{2}, 1\right) \oplus\left(\frac{3}{2}, 2\right) \oplus\left(\frac{1}{2}, 2\right) \oplus\left(\frac{3}{2}, 0\right) \oplus\left(\frac{1}{2}, 1\right)} \\
\quad \oplus\left(\frac{1}{2}, 0\right)\end{array}$ \\
\hline$\left\langle 1^{3} ; 1\right\rangle$ & 84 & $\begin{array}{l}{\left[\left(2, \frac{1}{2}\right) \oplus\left(0, \frac{3}{2}\right)\right] \Theta\left(\frac{1}{2}, 1\right) \cong\left(\frac{3}{2}, \frac{3}{2}\right) \oplus\left(\frac{3}{2}, \frac{1}{2}\right) \oplus\left(\frac{3}{2}, \frac{3}{2}\right) \oplus\left(\frac{3}{2}, \frac{1}{2}\right) \oplus\left(\frac{1}{2}, \frac{3}{2}\right) \oplus\left(\frac{1}{2}, \frac{3}{2}\right)} \\
\quad \oplus\left(\frac{1}{2}, \frac{1}{2}\right)\end{array}$ \\
\hline$\langle 2 ; 1\rangle$ & 126 & $\begin{array}{l}{[(2,1) \oplus(1,0) \oplus(0,1)] \Theta\left(\frac{1}{2}, 1\right) \cong\left(\frac{5}{2}, 2\right) \oplus\left(\frac{5}{2}, 1\right) \oplus\left(\frac{5}{2}, 0\right) \oplus\left(\frac{3}{2}, 2\right) \oplus 2\left(\frac{3}{2}, 1\right)} \\
\quad \oplus\left(\frac{3}{2}, 0\right) \oplus 2\left(\frac{1}{2}, 1\right) \oplus\left(\frac{1}{2}, 2\right) \oplus\left(\frac{1}{2}, 0\right)\end{array}$ \\
\hline$\langle 21 ; 1\rangle$ & 384 & $\begin{array}{l}{\left[\left(3, \frac{1}{2}\right) \oplus\left(2, \frac{3}{2}\right) \oplus\left(2, \frac{1}{2}\right) \oplus\left(1, \frac{3}{2}\right) \oplus\left(1, \frac{1}{2}\right) \oplus\left(0, \frac{1}{2}\right)\right] \otimes\left(\frac{1}{2}, 1\right) \cong\left(\frac{7}{2}, \frac{3}{2}\right) \oplus\left(\frac{3}{2}, \frac{1}{2}\right)} \\
\quad \oplus 3\left(\frac{3}{2}, \frac{3}{2}\right) \oplus 3\left(\frac{5}{2}, \frac{1}{2}\right) \oplus\left(\frac{3}{2}, \frac{3}{2}\right) \oplus 2\left(\frac{3}{2}, \frac{5}{2}\right) \oplus 4\left(\frac{3}{2}, \frac{3}{2}\right) \oplus 4\left(\frac{3}{2}, \frac{1}{2}\right) \oplus\left(\frac{1}{2}, \frac{5}{2}\right) \oplus 3\left(\frac{1}{2}, \frac{3}{2}\right) \\
\quad \oplus 3\left(\frac{1}{2}, \frac{1}{2}\right)\end{array}$ \\
\hline$\left\langle 1^{2} ; 1^{2}\right\rangle$ & 196 & $\begin{array}{l}{[(2,0) \oplus(1,1)] \otimes[(0,2) \oplus(1,1)] \cong 2(2,2) \oplus(3,1) \oplus(1,3) \oplus 2(2,1) \oplus 2(1,2)} \\
\quad \oplus 2(1,1) \oplus(2,0) \oplus(0,2) \oplus(1,1) \oplus(1,0) \oplus(0,1) \oplus(0,0)\end{array}$ \\
\hline$\left\langle 1^{3} ; 1^{2}\right\rangle$ & 196 & $\begin{array}{l}{\left[\left(2, \frac{1}{2}\right) \oplus\left(0, \frac{3}{2}\right)\right] \otimes[(0,2) \oplus(1,1)] \cong\left(2, \frac{5}{2}\right) \oplus 2\left(2, \frac{3}{2}\right) \oplus\left(3, \frac{3}{2}\right) \oplus\left(3, \frac{1}{2}\right) \oplus\left(2, \frac{1}{2}\right)} \\
\quad \oplus 2\left(1, \frac{3}{2}\right) \oplus 2\left(1, \frac{1}{2}\right) \oplus\left(1, \frac{5}{2}\right) \oplus\left(0, \frac{7}{2}\right) \oplus\left(0, \frac{5}{2}\right) \oplus\left(0, \frac{3}{2}\right) \oplus\left(0, \frac{1}{2}\right)\end{array}$ \\
\hline$\left\langle 1^{3} ; 1^{3}\right\rangle$ & 196 & $\begin{array}{l}{\left[\left(2, \frac{1}{2}\right) \oplus\left(0, \frac{3}{2}\right)\right] \otimes\left[\left(\frac{1}{2}, 2\right) \oplus\left(\frac{3}{2}, 0\right)\right] \cong\left(\frac{5}{2}, \frac{3}{2}\right) \oplus\left(\frac{5}{2}, \frac{3}{2}\right) \oplus\left(\frac{3}{2}, \frac{5}{2}\right) \oplus 2\left(\frac{3}{2}, \frac{3}{2}\right) \oplus\left(\frac{1}{2}, \frac{7}{2}\right)} \\
\quad \oplus\left(\frac{1}{2}, \frac{5}{2}\right) \oplus\left(\frac{1}{2}, \frac{3}{2}\right) \oplus 2\left(\frac{1}{2}, \frac{1}{2}\right) \oplus\left(\frac{3}{2}, \frac{1}{2}\right) \oplus\left(\frac{5}{2}, \frac{1}{2}\right) \oplus\left(\frac{3}{2}, \frac{1}{2}\right)\end{array}$ \\
\hline$\langle 2 ; 2\rangle$ & 441 & $\begin{array}{l}{[(2,1) \oplus(1,0) \oplus(0,1)] \otimes[(1,2) \oplus(1,0) \oplus(0,1)] \cong(3,3) \oplus(3,2) \oplus 2(3,1)} \\
\quad \oplus(2,3) \oplus 3(2,2) \oplus 3(2,1) \oplus 2(1,3) \oplus 3(1,2) \oplus 5(1,1) \oplus 2(0,2) \oplus 2(2,0) \\
\quad \oplus(1,0) \oplus(0,1) \oplus 2(0,0)\end{array}$ \\
\hline
\end{tabular}

In order to discuss the mass spectra and external field problem for this family of wave equations, we must consider the properties of the matrix $\alpha^{\mu} p_{\mu}$.

For the Kursunoğlu equation itself, it is convenient to enumerate the basis of $V_{1}$ as

$$
\left\{v_{m n}\right\}=\left\{v_{1, \frac{1}{2}}, v_{0, \frac{1}{2}}, v_{-1, \frac{1}{2}}, v_{1,-\frac{1}{2}}, v_{0,-\frac{1}{2}}, v_{-1,-\frac{1}{2}}\right\}
$$

We then take $\left\{v_{n m}\right\}$, ordered in the same way, as a basis for $V_{2}$. With respect to this basis we see that, from (3.5),

$$
B_{1}=B_{2}=i\left[\begin{array}{rrrrr} 
& & & & \\
& & & 1 & \\
& & -1 & & 0
\end{array}\right]
$$


By direct calculation, using (3.8) and (3.10), we find that the matrices $T^{A B}\left(a_{12}, a_{21}\right)$ are given by:

$$
\begin{aligned}
& T^{1 \mathrm{i}}=\left(\begin{array}{cc}
0 & a_{12} A^{1 \mathrm{i}} \\
-a_{21} A^{2 \dot{2}} & 0
\end{array}\right), T^{1 \dot{2}}=\left(\begin{array}{cc}
0 & a_{12} A^{1 \dot{2}} \\
a_{21} A^{1 \dot{2}} & 0
\end{array}\right), \\
& T^{2 \mathrm{i}}=\left(\begin{array}{cc}
0 & a_{12} A^{2 \mathrm{i}} \\
a_{21} A^{2 \mathrm{i}} & 0
\end{array}\right), T^{2 \dot{2}}=\left(\begin{array}{cc}
0 & a_{12} A^{2 \dot{2}} \\
-a_{21} A^{1 \mathrm{i}} & 0
\end{array}\right),
\end{aligned}
$$

where

$$
\begin{aligned}
& A^{1 \mathrm{i}}=\left[\begin{array}{rrrrrr}
0 & 0 & 0 & 0 & 0 & 0 \\
0 & 1 & 0 & 0 & 0 & 0 \\
0 & 0 & 0 & 0 & \sqrt{ } 2 & 0 \\
0 & 0 & 0 & 0 & 0 & 0 \\
0 & 0 & \sqrt{ } 2 & 0 & 0 & 0 \\
0 & 0 & 0 & 0 & 0 & 2
\end{array}\right], A^{1 \dot{2}}=\left[\begin{array}{rrrrrr}
0 & 0 & 0 & 0 & 0 & 0 \\
-\sqrt{ } 2 & 0 & 0 & 0 & 0 & 0 \\
0 & 0 & 0 & -2 & 0 & 0 \\
0 & 0 & 0 & 0 & 0 & 0 \\
0 & -1 & 0 & 0 & 0 & 0 \\
0 & 0 & 0 & 0 & -\sqrt{ } 2 & 0
\end{array}\right], \\
& A^{2 \mathrm{i}}=\left[\begin{array}{rrrrrr}
0 & -\sqrt{ } 2 & 0 & 0 & 0 & 0 \\
0 & 0 & 0 & 0 & -1 & 0 \\
0 & 0 & 0 & 0 & 0 & 0 \\
0 & 0 & -2 & 0 & 0 & 0 \\
0 & 0 & 0 & 0 & 0 & -\sqrt{ } 2 \\
0 & 0 & 0 & 0 & 0 & 0
\end{array}\right], A^{2 \dot{2}}=\left[\begin{array}{rrrrrr}
2 & 0 & 0 & 0 & 0 & 0 \\
0 & 0 & 0 & \sqrt{ } 2 & 0 & 0 \\
0 & 0 & 0 & 0 & 0 & 0 \\
0 & \sqrt{ } 2 & 0 & 0 & 0 & 0 \\
0 & 0 & 0 & 0 & 1 & 0 \\
0 & 0 & 0 & 0 & 0 & 0
\end{array}\right] \text {, }
\end{aligned}
$$

These matrices are skew relative to $B=B_{1} \oplus\left(a_{12} / a_{21}\right) B_{1}$.

We take our Cartan sub-algebra $H$ of $s p(12)$ to be the linear span of the matrices

$$
\begin{aligned}
& h_{1}=e_{11}-e_{66}, \\
& h_{2}=e_{22}-e_{55}, \\
& h_{3}=e_{33}-e_{44}, \\
& h_{4}=e_{77}-e_{12,12}, \\
& h_{5}=e_{88}-e_{11,11}, \\
& h_{6}=e_{99}-e_{10.10} .
\end{aligned}
$$


If $\varepsilon_{l}: H \rightarrow \mathrm{C}$ are the coordinate functions on $H$, then the simple roots are

$$
\begin{aligned}
& \alpha_{1}=\varepsilon_{1}-\varepsilon_{2}, \\
& \alpha_{2}=\varepsilon_{2}-\varepsilon_{3}, \\
& \alpha_{3}=\varepsilon_{3}-\varepsilon_{4}, \\
& \alpha_{4}=\varepsilon_{4}-\varepsilon_{5}, \\
& \alpha_{5}=\varepsilon_{5}-\varepsilon_{6}, \\
& \alpha_{6}=2 \varepsilon_{6},
\end{aligned}
$$

and so the fundamental dominant weights are

$$
\lambda_{i}=\sum_{j=1}^{i} \varepsilon_{j}, \quad 1 \leqslant i \leqslant 6 .
$$

To simplify the calculation, we shall take $a_{12}=-a_{21}$ to be real, thus ensuring that the Kursunoğlu equation is invariant under spatial reflection and may be derived from an invariant Lagrangian (see (4.6) and (4.33)). By changing $\kappa$ if necessary, we may as well assume that $a_{12}=-a_{21}=1$. By (5.8) we see that the minimal and characteristic polynomials of $\alpha^{\mu} p_{\mu}$ are:

$$
\begin{aligned}
\min \left(\alpha^{\mu} p_{\mu}\right) & =\left(x^{2}-4 p^{2}\right)\left(x^{2}-p^{2}\right), \\
\operatorname{ch}\left(\alpha^{\mu} p_{\mu}\right) & =\left(x^{2}-4 p^{2}\right)^{4}\left(x^{2}-p^{2}\right)^{2}
\end{aligned}
$$

(where $p^{2}=p_{\mu} p^{\mu}=\left(p^{0}\right)^{2}-\mathbf{p}^{2}$ ).

Thus, if $p^{2} \neq 0, \alpha^{\mu} p_{\mu}$ is semi-simple, and so it lies in some CSA $K$ of $s p(12)$ ([15], p. 35). We know then that there is an inner automorphism $\eta_{p}$ of $s p(12)$ such that $\eta_{p}(K)=H$. Such an automorphism is of the form:

$$
\begin{gathered}
\eta_{p}(X)=O(p)^{-1} X O(p), \quad X \in s p(12), \\
O(p)^{\mathrm{T}} B O(p)=B
\end{gathered}
$$

(see, for example, [22], p. 284). The matrix $\eta_{p}\left(\alpha^{\mu} p_{\mu}\right) \in H$ is diagonal, its diagonal elements being the eigenvalues of $\alpha^{\mu} p_{\mu}$ in some order. Now we demand further that, when $p=\hat{p}=(1,0,0,0)$ (that is $\left.\alpha^{\mu} p_{\mu}=\alpha^{0}\right)$, the map $\eta_{\hat{p}}$ does not alter the embedding $s o(3) \subset s p(12)$, where $s o(3)$ denotes the diagonal sub-algebra of $s o(4) \cong s /(2) \oplus s /(2)$. We therefore assume that

$$
\eta_{\hat{p}}\left(H_{3}\right)=O(\hat{p})^{-1} H_{3} O(\hat{p})=H_{3}
$$

where

$$
H_{3}=K_{3}+L_{3}=\operatorname{diag}\left\{\frac{3}{2}, \frac{1}{2},-\frac{1}{2}, \frac{1}{2},-\frac{1}{2},-\frac{3}{2}, \frac{3}{2}, \frac{1}{2}-\frac{1}{2}, \frac{1}{2},-\frac{1}{2},-\frac{3}{2}\right\} .
$$

By direct calculation, we find that 
480

A. Cant and C. A. Hurst

[35]

$$
\begin{aligned}
& 0 \quad 0 \quad 0 \quad 0 \quad 0 \sum_{i}^{i} 000000 \sum_{i}^{i} \\
& \text { - } 0 \sum_{i}^{i} 0 \sum_{i}^{i} 0000 \frac{i}{j} 0 \sum_{i}^{i} 0 \\
& \text { - } i_{i}^{i} \circ \sum_{i}^{i} 000 i_{i}^{i} 0 i_{i}^{i} 00 \\
& 00 \sum_{i}^{i} 0 i_{i}^{i} 000 \sum_{i}^{i} 0 i_{0}^{i} 0 \\
& \text { - } i_{i}^{i} \circ \sum_{i}^{i} 000 \sum_{i}^{i} 0 i_{i}^{i} 00
\end{aligned}
$$

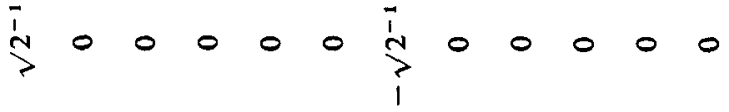

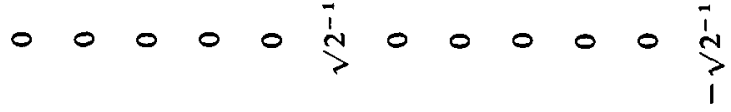

$$
\begin{aligned}
& 00 i_{i}^{i} 0 i_{i}^{i} 000 i_{i}^{i} 0 \sum_{i}^{i} 0 \\
& 0 i_{i}^{i} 0 i_{i}^{i} 000 \sum_{i}^{i} 0 j_{i}^{i} 00
\end{aligned}
$$

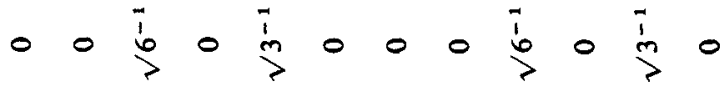

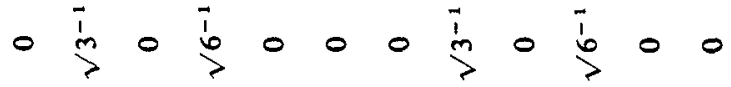

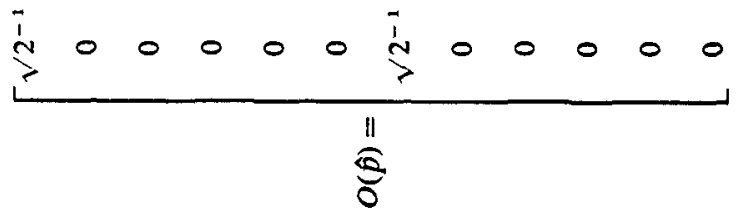

https://doi.org/10.1017/S1446181100001802 Published online by Cambridge University Press 
This now fixes the order of the diagonal elements of $\eta_{p}\left(\alpha^{\mu} p_{\mu}\right)$, and we have

$$
\eta_{p}\left(\alpha^{\mu} p_{\mu}\right)=\left(2 h_{1}+2 h_{2}+2 h_{3}-2 h_{4}+h_{5}+h_{6}\right)\left(p^{2}\right)^{\frac{1}{2}} .
$$

We now go over to an irreducible representation $\left(\pi_{\lambda}, V(\lambda)\right)$ of $s p(12)$ with highest weight

$$
\lambda=\sum_{i=1}^{6} m_{i} \lambda_{i} .
$$

It is clear that the characteristic polynomial of $\pi_{\lambda}\left(\eta_{p}\left(\alpha^{\mu} p_{\mu}\right)\right.$ ) (and hence of $\pi_{\lambda}\left(\alpha^{\mu} p_{\mu}\right)$ ) will be

$$
\prod_{\nu}\left(x-v\left(\eta_{D}\left(\alpha^{\mu} p_{\mu}\right)\right)\right)
$$

the product being taken over all weights $v$ of $\pi_{\lambda}$. If we write $v=\sum \quad n_{i} \lambda_{i}$, and use (5.11), then the characteristic polynomial of $\pi_{\lambda}\left(\alpha^{\mu} p_{\mu}\right)$ becomes

$$
\prod_{v=\Sigma n_{n_{1} \lambda_{i}}}\left(x-G(v)\left(p^{2}\right)^{\frac{1}{2}}\right)
$$

(valid now for any $p_{\mu}$ ), where

$$
G(v)=2 n_{1}+4 n_{2}+6 n_{3}+4 n_{4}+5 n_{5}+6 n_{6} .
$$

In particular, (5.13) tells us that $\pi_{\lambda}\left(\alpha^{\mu} p_{\mu}\right)$ has exactly as many zero eigenvalues as there are weights $v$ of $\pi_{\lambda}$ for which $G(v)=0$. Now the irreducible representation $\pi_{\lambda}$ of $s p(12)$ is one of two kinds; if $m_{1}+m_{3}+m_{5}$ is even, it is orthogonal (integral spin), and if $m_{1}+m_{3}+m_{s}$ is odd, it is symplectic (half-integral spin). In the former case, we observe that $\lambda$ is a sum of roots, and so 0 is a weight of $\pi_{\lambda}$. Since $G(0)=0$, we see that $\pi_{\lambda}\left(\alpha^{\mu} p_{\mu}\right)$ will always be singular for integral spin equations. In the latter case, $\pi_{\lambda}\left(\alpha^{\mu} p_{\mu}\right)$ may or may not be singular.

These results should be contrasted with the corresponding properties of the $s o(5)$ equations, where $\alpha^{\mu} p_{\mu}$ is always singular for integral spin, and always nonsingular for half-integral spin [23].

From the above we may immediately write down the spectrum of rest masses for the wave equation corresponding to $\pi_{\lambda}$. By going to the rest frame $p=0$ in (1.1), we see that $p_{0}$ has the possible values

$$
p_{\mathrm{o}}=\frac{\kappa}{G(v)} \text { for all } v \text { such that } G(v) \neq 0 .
$$

We exclude, therefore, the "states" in the null space of $\alpha^{0}$ since they would correspond to (unphysical) infinite masses. This null space is invariant under so(3), and so the corresponding spins will have to be excluded. They may easily be found by applying the map $f^{*}$ associated with the embedding $f: s o(3) \rightarrow \operatorname{sp}(12)$. The situation is analogous to that for the $s o(5)$ equations, where, for example, in the five-dimensional representation the states corresponding to spin 1 are unphysical [5]. 
We conclude with some brief remarks on the causality properties of these equations, with or without an external field being present. We refer the reader to [23] for a thorough general discussion of this problem, and an analysis of the causality properties of the $s o(5)$ equations.

If $\pi_{\lambda}\left(\alpha^{\mu} p_{\mu}\right)$ is non-singular, then the corresponding wave equation is hyperbolic (in the sense of Courant-Hilbert [24]). We know then that there exists a retarded fundamental solution, and so propagation is causal. This remains true even in the presence of an external field, provided we assume minimal coupling:

$$
\partial_{\mu} \rightarrow \partial_{\mu}^{-}=\partial_{\mu}-i e A_{\mu} \quad\left(p_{\mu} \rightarrow p_{\mu}^{-}=p_{\mu}+e A_{\mu}\right) .
$$

The situation is more complicated if $\pi_{\lambda}\left(\alpha^{\mu} p_{\mu}\right)$ happens to be singular, because the wave equation is then not hyperbolic as it stands. In this case, the trick is to study the associated multi-mass Klein-Gordon equation [23]:

$$
d(\partial) \Lambda(\partial) \psi(x)=\prod_{i=1}^{r}\left(\square-\frac{\kappa^{2}}{b_{i}^{2}}\right) \psi(x)=0,
$$

where $\Lambda(\partial)=\alpha^{\mu} \partial_{\mu}+i \kappa, \square=\partial_{\mu} \partial^{\mu}$ and $\pm b_{1}, \ldots, \pm b_{r}$ are the non-zero eigenvalues of $\alpha^{0}$ (for brevity, we write $\alpha^{\mu}$ now instead of $\pi_{\dot{\lambda}}\left(\alpha^{\mu}\right)$ ). The operator $d(\partial)$ is called the Klein-Gordon divisor; it exists because $\operatorname{det}\left(\alpha^{\mu} \partial_{\mu}+i \kappa\right)$ does not vanish identically. The equation (5.14) is certainly hyperbolic. To study the effect of an external field, we must find the order of the highest derivative term in $d(\partial)$.

Going over to momentum space, if 0 is an $s$-fold eigenvalue of $\alpha^{0}$, we know from above that $\alpha^{\mu} p_{\mu}-\kappa$ is similar to the matrix

$$
\operatorname{diag}\{\kappa-b_{1} \sqrt{ } p^{2}, \kappa+b_{1} \sqrt{ } p^{2}, \ldots, \kappa-b_{r} \sqrt{ } p^{2}, \kappa+b_{r} \sqrt{ } p^{2}, \underbrace{\kappa, \ldots, \kappa}_{\text {sfactors }}\} .
$$

Thus $\left(\alpha^{\mu} p_{\mu}-\kappa\right)^{-1}$ will have highest power zero in $p$ (not -1$)$. Therefore, $d(p)$ has the highest power $2 r$ in $p$, the same as the operator

$$
\prod_{i=1}^{r}\left(p^{2}-\frac{\kappa^{2}}{b_{i}^{2}}\right)
$$

We now see that, if we use

$$
\begin{aligned}
& {\left[\partial_{\mu}^{-}, \partial_{v}^{-}\right]=-i e F_{\mu v}\left(F_{\mu v}=\partial_{\mu} A_{v}-\partial_{v} A_{\mu}\right):} \\
& d\left(\partial^{-}\right) \Lambda\left(\partial^{-}\right)=\prod_{i=1}^{r}\left(\partial^{-} \partial^{-}-\frac{\kappa^{2}}{b_{i}^{2}}\right)+B(\partial),
\end{aligned}
$$

where $B(\partial)$ has highest derivatives of order $2 r-1$. By the same argument as in [23], we can conclude that propagation is causal for the coupled equations.

In fact, a similar argument goes through for any wave equation of the form (1.1). Problems with causality seem to be mainly associated with those wave equations having subsidiary conditions [24]. 


\section{Conclusions. Suggestions for further work}

The main point of this paper has been to show that the $s o(5)$ family of wave equations is merely one possibility: as we have seen, there are an enormous number of other equations, including those associated with the symplectic and orthogonal Lie algebras. Our aim was not to discuss explicitly every possible wave equation, but to indicate some general properties. It is ultimately a matter of taste and further physical requirements which dictates the kinds of wave equations to allow. Current work on wave equations has focused attention on the problems of second quantization, causality and the appearance of negative probabilities. Some comments and many recent references are given in $[23,30]$; in particular it is concluded in [30] that for the so(5) equation there is a built-in or "kinematic" indefinite metric, and it is hard to remove this feature in a quantized picture.

There are two further topics arising from this paper which are worth investigation. Firstly, one might consider the infinite-dimensional metaplectic representations of the appropriate real forms of $s p(n)$ and $s o(n)$ as possible wave equations. These would be analogous to the Majorana equations, which correspond to the so-called "ladder" representations of $s p(4, \mathbf{R})$ [25]. Secondly, there is the problem of describing zero mass wave equations, which are more general than massive wave equations in the sense that the invariance condition (1.3) now reads

$$
\rho^{-1}(\Lambda) \alpha^{\mu} \pi(\Lambda)=\Lambda_{\gamma}^{\mu} \alpha^{\nu}
$$

where $\rho, \pi$ are arbitrary representations of $s o(4)[8]$. Thus the matrices $\alpha^{\mu}$ may in general be rectangular.

\section{Acknowledgements}

One of us (C. A. H.) would like to thank Professor Behram Kursunoğlu for first introducing him to the Kursunoğlu equation and for his hospitality at the Center for Theoretical Studies, Coral Gables, where these ideas were first developed.

Both authors would like to thank Dr. A. L. Carey for many helpful suggestions. We are also grateful for discussions with Prof. H. S. Green, Prof. K. J. Le Couteur, Prof. L. Simon, Dr. J. F. Cornwell, Dr. M. Vujičić and J. W. Evans.

The receipt of a Commonwealth Postgraduate Research Award by A. C. is gratefully acknowledged.

\section{Appendix}

We discuss here the problem of calculating the multiplicity of a weight in an irreducible $C_{3}$-module. One way which is quite rapid is to use a character formula derived recently by Demazure [26]. For modules of large dimension, Kostant's formula is perhaps the most useful, since the Weyl group $W$ of $C_{3}$ has order 48 , 
which is not too unwieldy. This formula involves the "partition function" for $C_{3}$, which does not seem to have been given explicitly elsewhere, so we now derive an expression for it.

Recall that if $L$ is a complex semi-simple Lie algebra, with $H$ a Cartan subalgebra, then the multiplicity $m_{\lambda}(\mu)$ of the weight $\mu \in H^{*}$ in the irreducible $L$-module $V(\lambda)$ is given by Kostant's formula (see, for example, [15], p. 138):

$$
m_{\lambda}(\mu)=\sum_{\sigma \in W} \operatorname{sn}(\sigma) P_{L}(\sigma(\lambda+\delta)-(\mu+\delta))
$$

Here, for $\sigma \in W, \operatorname{sn}(\sigma)=+1$ or -1 according as $\sigma$ is the product of an even or odd number of simple reflections $\sigma_{\alpha_{i}}=\sigma_{i}$, and $\delta=\frac{1}{2} \sum_{v>0} \alpha$. For $v \in H^{*}, P_{L}(v)$ is defined to be the number of sets of non-negative integers $\left\{k_{\alpha} ; \alpha>0\right\}$ such that

$$
v=\sum_{\alpha<0} k_{\alpha} \cdot \alpha
$$

and $P_{L}$ is called the partition function for $L$. Clearly $P_{L}(v)=0$ unless $v$ lies in the root lattice.

Tarski [27] found explicit formulae for $P_{L}$ where $L$ is $A_{1}, A_{2}, A_{3}, B_{2}$ or $G_{2}$, but his geometric method does not easily generalize to other Lie algebras. We can calculate $P_{L}$ for the case $L=C_{3}$ as follows.

The positive roots for $C_{3}$ are

$$
\begin{aligned}
\Phi^{+} & =\left\{\alpha_{1}, \alpha_{2}, \alpha_{3}, \alpha_{1}+\alpha_{2}, \alpha_{2}+\alpha_{3}, \alpha_{1}+\alpha_{2}+\alpha_{3}, 2 \alpha_{2}+\alpha_{3}, \alpha_{1}+2 \alpha_{2}+\alpha_{3}, 2 \alpha_{1}+2 \alpha_{2}+\alpha_{3}\right\} \\
& =\left\{\beta_{1}, \ldots, \beta_{9}\right\}
\end{aligned}
$$

with simple roots $\Delta=\left\{\alpha_{1}, \alpha_{2}, \alpha_{3}\right\}$. If $v \in H^{*}$, write $v=n_{1} \alpha_{1}+n_{2} \alpha_{2}+n_{3} \alpha_{3}$. Then the condition

$$
v=\sum_{i=1}^{9} k_{i} \beta_{r}
$$

gives the following system of Diophantine equations:

$$
\begin{aligned}
& n_{1}=k_{1}+k_{4}+k_{6}+k_{8}+2 k_{9}, \\
& n_{2}=k_{2}+k_{4}+k_{5}+k_{6}+2 k_{7}+2 k_{8}+2 k_{9}, \\
& n_{3}=k_{3}+k_{5}+k_{6}+k_{7}+k_{8}+k_{9}
\end{aligned}
$$

which we write as

$$
\begin{gathered}
n_{1}-k_{8}-2 k_{9}=k_{1}+k_{4}+k_{6}, \\
n_{2}-2 k_{7}-2 k_{8}-2 k_{9}=k_{2}+k_{4}+k_{5}+k_{6}, \\
n_{3}-k_{7}-k_{8}-k_{9}=k_{3}+k_{5}+k_{6} .
\end{gathered}
$$

The latter equations effectively reduce the problem to the situation $L=A_{3}$, already solved by Gruber and Zaccaria ([28], p. 928). This is clear because

The method of generating functions leads to a similar formula for the partition function [31]. 
the positive roots for $A_{3}$ are $\left\{\alpha_{1}^{\prime}, \alpha_{2}^{\prime}, \alpha_{3}^{\prime}, \alpha_{1}^{\prime}+\alpha_{2}^{\prime}, \alpha_{2}^{\prime}+\alpha_{3}^{\prime}, \alpha_{1}^{\prime}+\alpha_{2}^{\prime}+\alpha_{3}^{\prime}\right\}$, with $\alpha_{1}^{\prime}, \alpha_{2}^{\prime}, \alpha_{3}^{\prime}$ being the simple roots of $A_{3}$. Thus, as far as combinatorial properties are concerned, the positive root system for $A_{3}$ behaves like the subset $\left\{\beta_{1}, \ldots, \beta_{6}\right\}$ of $\Phi^{+}$. We therefore have

$$
\begin{aligned}
P_{C_{3}}(v) & \equiv P_{C_{3}}\left(n_{1}, n_{2}, n_{3}\right) \\
& =\sum_{i \geqslant 0} \sum_{j \geqslant 0} \sum_{k \geqslant 0} P_{A_{3}}\left(n_{1}-j-2 k, n_{2}-2(i+j+k), n_{3}-(i+j+k)\right),
\end{aligned}
$$

where $P_{A_{3}}$ is given in terms of $P_{A_{2}}$ by means of the formula [28]:

$$
\begin{array}{rlr}
P_{A_{3}}\left(n_{1}, n_{2}, n_{3}\right) & =\sum_{i=0}^{n_{1}} \sum_{j=0}^{n_{2}-i} P_{A_{2}}\left(n_{1}-i, n_{2}-i-j\right) & \text { if } n_{1}, n_{2} \leqslant n_{3} \\
& =\sum_{i=0}^{n_{3}} \sum_{j=0}^{n_{1}-i} P_{A_{2}}\left(n_{2}-i-j, n_{3}-i\right) & \text { if } n_{3} \leqslant n_{1} \leqslant n_{2} \\
& =\sum_{i=0}^{n_{3}} \sum_{j=0}^{n_{2}-i} P_{A_{2}}\left(n_{3}-i, n_{2}-i-j\right) & \text { if } n_{3}, n_{2} \leqslant n_{1} \\
& =\sum_{i=0}^{n_{1}} \sum_{j=0}^{n_{3}-i} P_{A_{2}}\left(n_{2}-i-j, n_{1}-i\right) & \text { if } n_{1} \leqslant n_{3} \leqslant n_{2} .
\end{array}
$$

Finally, the partition function for $A_{2}$ is given by

$$
P_{A}\left(s_{2}, s_{2}\right)= \begin{cases}1+\min \left(s_{1}, s_{2}\right) & \text { if } s_{1}, s_{2} \text { are non-negative integers, } \\ 0 & \text { otherwise. }\end{cases}
$$

Thus we have obtained an explicit although rather complicated formula for the partition function for $C_{3}$, and we may use Kostant's formula to find the multiplicity of a weight in an irreducible $C_{3}$-module.

\section{References}

[1] P. A. M. Dirac, Proc. Roy. Soc. (London) A 115 (1936), 447-459.

[2] M. Fierz, Helv. Phys. Acta 12 (1939), 3-37.

W. Pauli and M. Fierz, Helv. Phys. Acta 12 (1939), 297-300.

M. Fierz and W. Pauli, Proc. Roy. Soc. A 173 (1939), 211-232.

[3] H. J. Bhabha, Rev. Mod. Phys. 17 (1945), 200-216.

[4] Harish-Chandra, Phys. Rev. 71 (1947), 793-805.

[5] K. J. Le Couteur, Proc. Roy. Soc. (London) A 202 (1950), 284-300, 394-407.

[6] I. M. Gel'fand and A. M. Yaglom, Ž. Ėksper. Teoret. Fiz. 18 (1948), 703-733, 1096-1104, $1105-1111$.

[7] E. M. Corson, An introduction to tensors, spinors and relativistic wave equations (Blackie, 1953).

[8] I. M. Gel'fand, R. A. Minlos and Z. Ya. Shapiro, Representations of the rotation and Lorentz groups and their applications (Pergamon Press, 1963).

[9] F. L. Bauer, Sitzb. d. Bay. Akad. d. Wiss. 13 (1952), 111-179.

[10] M. Lorente, P. L. Huddleston and P. Roman, J. Math. Phys. 14 (1973), 1495-1497. 
[11] H. Biritz, Nuov. Cim. B 25 (1975), 449-478.

[12] E. B. Dynkin, Amer. Math. Soc. Transl. (Ser. 2) 6 (1957), 111-244.

[13] B. Kursunoğlu, Phys. Rev. 167 (1968), 1452-1461.

[14] A. Navon and J. Patera, J. Math. Phys. 8 (1967), 489-493.

[15] J. E. Humphreys, Introduction to Lie algebras and representation theory (Springer-Verlag, 1972).

[16] R. C. King, J. Phys. A 8 (1975), 429-449.

[17] H. Samelson, Notes on Lie algebras (Van Nostrand Reinhold, 1969).

[18] A. S. Wightman, "Stability of representations of the Poincaré group", in Symmetry principles at high energy, Fifth Coral Gables Conference (ed. A. Perlmutter, C. A. Hurst and B. Kursunoğlu) (W. A. Benjamin, 1968).

[19] B. G. Wybourne, Symmetry principles and atomic spectroscopy, (Wiley-Interscience, 1970).

[20] A. P. Yutsis, I. B. Levinson and V. V. Vanagas, Theory of angular momentum, (Israel Program for Scientific Translations, 1962).

[21] M. Konuma, K. Shima and M. Wada, Progr. Theoret. Phys. (Japan) Suppl. 28 (1963), 1-127.

[22] N. Jacobson, Lie algebras (Interscience, 1962).

[23] R. A. Krajcik and M. M. Nieto, Phys. Rev. D 13 (1976), 924-941.

[24] G. Velo and D. Zwanziger, "Acausality and higher spin equations", in Tracts in mathematics and natural sciences Vol. 4 (Gordon and Breach, 1971).

[25] D. Tz. Stoyanov and I. T. Todorov, J. Math. Phys. 9 (1968), 2146-2167.

[26] M. Demazure, Bull. Sci. Math. 2e série 98 (1974), 163-172.

[27] J. Tarski, J. Math. Phys. 4 (1963), 569-574.

[28] B. Gruber and F. Zaccaria, Suppl. al Nuovo Cimento 5 (1967), 914-936.

[29] A. J. Bracken, J. Phys. A 8 (1975), 800-807.

[30] R. A. Krajcik and M. M. Nieto, Phys. Rev. D 14 (1976), 418-436.

[31] T. S. Santhanam, J. Math. Phys. 10 (1969), 1704-1710.

Department of Mathematical Physics

University of Adelaide

Adelaide, South Australia, 5001 